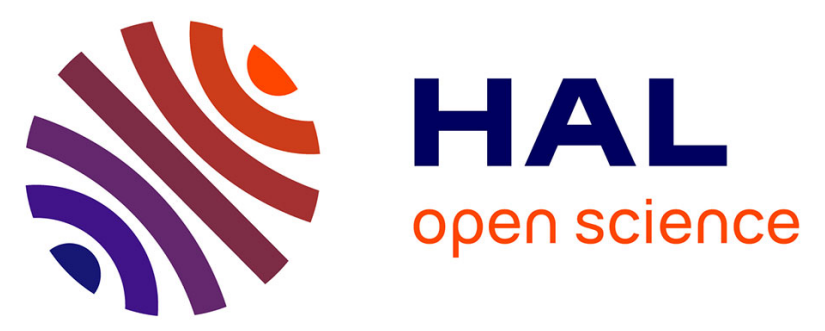

\title{
Looking for new antiplasmodial quinazolines: DMAP-catalyzed synthesis of 4-benzyloxy- and 4-aryloxy-2-trichloromethylquinazolines and their in vitro evaluation toward Plasmodium falciparum
}

Armand Gellis, Nicolas Primas, Sébastien Hutter, Gilles Lanzada, Vincent Remusat, Pierre Verhaeghe, Patrice Vanelle, Nadine Azas

\section{To cite this version:}

Armand Gellis, Nicolas Primas, Sébastien Hutter, Gilles Lanzada, Vincent Remusat, et al.. Looking for new antiplasmodial quinazolines: DMAP-catalyzed synthesis of 4-benzyloxy- and 4-aryloxy-2trichloromethylquinazolines and their in vitro evaluation toward Plasmodium falciparum. European Journal of Medicinal Chemistry, 2016, 119, pp.34-44. 10.1016/j.ejmech.2016.04.059 . hal-01417007

\author{
HAL Id: hal-01417007 \\ https://hal.science/hal-01417007
}

Submitted on 15 Dec 2016

HAL is a multi-disciplinary open access archive for the deposit and dissemination of scientific research documents, whether they are published or not. The documents may come from teaching and research institutions in France or abroad, or from public or private research centers.
L'archive ouverte pluridisciplinaire HAL, est destinée au dépôt et à la diffusion de documents scientifiques de niveau recherche, publiés ou non, émanant des établissements d'enseignement et de recherche français ou étrangers, des laboratoires publics ou privés. 


\section{Looking for new antiplasmodial quinazolines: DMAP-catalyzed synthesis of4-benzyloxy- and 4-aryloxy-2-trichloromethylquinazolines and their in vitro evaluation toward Plasmodium falciparum}

Armand Gellis ${ }^{1}$, Nicolas Primas ${ }^{1}$, Sébastien Hutter ${ }^{2}$, Gilles Lanzada ${ }^{1}$,Vincent Remusat ${ }^{1}$, Pierre Verhaeghe $^{3 *}$,Patrice Vanelle ${ }^{1}$ and Nadine Azas ${ }^{2}$.

${ }^{1}$ Aix-Marseille Université, CNRS, ICR UMR 7273, Laboratoire de Pharmaco-Chimie Radicalaire,Faculté de Pharmacie, 27 Boulevard Jean Moulin - CS30064, 13385 Marseille cedex 05, France.

${ }^{2}$ Aix-Marseille Université, UMR MD 3, Infections Parasitaires, Transmission et Thérapeutique, Faculté de Pharmacie, 27 Boulevard Jean Moulin - CS30064, 13385 Marseille cedex 05, France.

${ }^{3}$ Université Paul Sabatier, Faculté des Sciences Pharmaceutiques - CNRS UPR 8241, Laboratoire de Chimie de Coordination, 205 Route de Narbonne, 31077 Toulouse cedex 04, France.

*Corresponding author: Pierre Verhaeghe, Tel.: +33561333236; E-mail: pierre.verhaeghe@univ-tlse3.fr.

Abstract:A DMAP catalyzedsynthesis of new 4-benzyloxy- and 4-aryloxy-2trichloromethylquinazolines was studied, in a view to react 4-chloroquinazolines with poorly nucleophilic alcohols such as benzylic alcohols, via a simple and cheapS $\mathrm{S}_{\mathrm{N}} \mathrm{Ar}$ reaction approach. A fast $(1 \mathrm{~h})$ general operating procedure, affording good reaction yields, was achieved under microwave irradiation. Thus, a series of 35 molecules was obtained and evaluated in vitro on the $\mathrm{K} 1$ multi-resistant $P$. falciparum strain, in parallel with a cytotoxicity assessment on the human HepG2 cell line. 5 hit-molecules were identified, presenting both promising antiplasmodial activity $\left(1.5 \mu \mathrm{M}<\mathrm{IC}_{50}<2 \mu \mathrm{M}\right)$ and low cytotoxicities $(25 \mu \mathrm{M}<$ $\mathrm{CC}_{50}<45 \mu \mathrm{M}$ ). Apart for 2 molecules, the global series displayed a satisfying solubility in the aqueous biological media. Structure-activity relationships showed that the molecules presenting a benzyloxy moiety were less cytotoxic than the ones bearing a phenoxy moiety at position 4 of the quinazoline ring. It also appeared that the introduction of a heteroaryl moiety afforded inactive compounds. Finally, the most active and selective molecules (Selectivity index $=22-27$ ) were the ones presenting either an unsubstituted benzyloxy group or a phenoxy group, this last bearing a $p$-bromo or an $o$-acetyl substituent.

Highlights: The DMAP-catalyzed $\quad \mathrm{S}_{\mathrm{N}} \mathrm{Ar}$ reactionbetween 4-chloro-2trichloromethylquinazoline and various alcohols or phenols was studied. A series of 35 new quinazolineswas synthesized in good yields. In vitroevaluationwas made on Plasmodium falciparum. 5 selective antiplasmodial hit molecules were identified.

Keywords:2-trichloromethylquinazoline; 4-benzyloxyquinazoline; 4-phenoxyquinazoline; DMAP-catalysis, $\mathrm{S}_{\mathrm{N}} \mathrm{Ar}$ reaction, microwave-assisted chemistry, Plasmodium falciparum; antiplasmodial activity;HepG2 cytotoxicity, SARs 


\section{Introduction}

Plasmodium falciparum is the protozoa responsible for cerebral malaria, the leading cause of death among parasitic infections worldwide. According to the 2015 World Malaria Report [1], 214 million people were infected byPlasmodium in 2014. It was also estimated that 438.000 people died from this parasitic infection, mainly because of the cerebral form of the disease. About $88 \%$ of the estimated deaths occurred in Africa, children under five representing a large majority of the victims.

Since the beginning of 2000 , a very significant improvement of the situation has been reached, thanks to the WHO intervention and the involvement of several non-governmental organizations (Bill and Melinda Gates Foundation, Roll Back Malaria, Medicines for Malaria Venture...).

Nevertheless, the emergence of drug-resistant strains of the parasite in Africa, South-East Asia and South America remains a serious concern for the medical and scientific community, in a view to keep on controlling the infection and to try to eradicate the disease. For that purpose, the treatment of the patients infected by $P$. falciparum is based on combination therapies including artemisinin derivatives, in order to cure the disease and avoid the selection or resistant strains and their spreading in the exposed population, which would lead to a very worrying sanitary situation. Concerning artemisinin derivatives, which are nowadays keymolecules for the treatment of $P$. falciparum malaria, resistances are emerging in Asia [2], and it has been demonstrated that they areresponsible for therapeutic failures in several infected patients [3]. Moreover, it has also recently been highlighted that the African Anopheles gambiae mosquito could transmit such Asian resistant parasites [4], indicating a major worldwide spreading risk. Thus, research efforts have to be maintained so as to discover new chemical entities presenting novel antiplasmodial mechanisms of action, to use in combination therapies with the existing antimalarial drugs, to guaranty their durable efficiency.

Considering all the scaffolds which were studied for their antiplasmodial potential, several bioactive molecules are based on a quinazolinering. For example, febrifugine is natural alkaloidincluding a quinazolinone moiety which isextracted from the Chinese herb Dichroafebrifuga, and which was employed by local people as a medicine against fevers caused by malaria. Some synthetic febrifugine derivatives were prepared and displayed good in vitro and in vivo potential [5]. Many other quinazoline derivatives were synthesized and evaluated toward P. falciparum, in particular, quinazolines bearing, an amino group [6], an alkylamine moiety [7,8] or an anilino substituent [9-11] at position 4 of the quinazoline ring.

In continuation with our research activity focusing on the synthesis of new nitrogencontaining heterocycles with anti-infective potential [12-14], our research group previously reported the synthesis and in vitro study ofnumerous antiplasmodial molecules based on a 2trichloromethylquinazoline scaffold. Thus, several series bearing ananilino [10], aryl [15], thiophenol [16], sulfonamide [17] or alkynyl moiety [18] at position 4 of the quinazoline ring were prepared and revealed several hit-molecules which are presented in Table 1. Among the antiplasmodial hit-molecules which were identified, a 4-phenoxy-2trichloromethylquinazoline derivative was also discovered [19], presenting a 50\% inhibitory concentration $\left(\mathrm{IC}_{50}\right)$ of $1.1 \mu \mathrm{M}$ and a $50 \%$ cytotoxicity concentration $\left(\mathrm{CC}_{50}\right)$ of $50 \mu \mathrm{M}$ (Table 
1), reaching an encouraging selectivity index of 45 , in comparison with chloroquine and doxycycline. Then, searching for novel analogs presenting an oxygen-containing substituent at position 4 of the quinazoline ring, we present herein the synthesis work which was conducted for reacting 4-chloro-2-trichloromethylquinazoline with poorly nucleophilic benzylic alcohols, to afford the target molecules in a fast, simple and cheap way, using the $\mathrm{S}_{\mathrm{N}} \mathrm{Ar}$ reaction. The in vitro biological evaluation and the SARs will then be presented and discussed.

Table 1. In vitro antiplasmodial profiles of the previously identified hit-molecules in 2trichloromethylquinazoline series

\begin{tabular}{|c|c|c|c|c|}
\hline $\begin{array}{c}\text { Antiplasmodial Activity } \\
\text { P. falciparum } W 2 \text { or } K 1 I_{50}(\mu M)\end{array}$ & 2.5 & 0.4 & 1.1 & 0.9 \\
\hline $\begin{array}{c}\text { Cytotoxicity } \\
H e p G 2 C C_{50}(\mu M)\end{array}$ & $>125$ & 16 & 50 & $>25$ \\
\hline $\begin{array}{l}\text { Selectivity Index } \\
\quad\left(C C_{50} / I C_{50}\right)\end{array}$ & 50 & 40 & 45 & $>28$ \\
\hline
\end{tabular}

Reference molecules: Chloroquine $\left(\mathrm{IC}_{50} \mathrm{~K} 1 P\right.$. falciparum $=0.6 \mu \mathrm{M}, \mathrm{CC}_{50}$ HepG2 $\left.=30 \mu \mathrm{M}, \mathrm{SI}=50\right)$; Doxycycline $\left(\mathrm{IC}_{50} \mathrm{~K} 1 \mathrm{P}\right.$. falciparum $=6 \mu \mathrm{M}, \mathrm{CC}_{50} \mathrm{HepG} 2=20 \mu \mathrm{M}, \mathrm{SI}=3.3$ )

\section{Results and discussion}

\subsection{Synthesis}

As aliphatic alcohols display poor nucleophilicity, reacting them with chlorinated azaheterocycles, to provide the corresponding $S_{N} A r$ products, is difficult and usually requires the preliminary in situ formation of the correspondingalcoholate anion. Thus, in quinazoline series, various operating procedures were reported in the literature, using methylate sodium in methanol [20], sodium hydride in dry DMF [21], sodium hydride in dry THF [22,23] or sodium in dry THF [24]. These protocols, leading to the expected products in moderate to good yields (32-84\%), present several disadvantages as they consist in 2 step-reactions (formation of the anion followed by the $S_{N} A r$ ), require the use of perfectly dry organic solvents and involve highly reactive and flammable reagents. Moreover, we tried to react 4chloro-2-trichloromethylquinazolinewith benzyl alcohol, using the reaction conditions we previously reported for the synthesis of 4-phenoxyquinazolines [19] (NaH, DMSO, RT, $24 \mathrm{~h}$ ) and obtained the expected product in a very low $11 \%$ yield (Table 2, entry 13).

Among the available alternative strategies, palladium cross-coupling reactions could have been considered. Nevertheless, because of the expensive costs of both palladium catalysts and ligands, in order to set up a cheap and simple operating procedure, we decided to study the one pot $\mathrm{S}_{\mathrm{N}} \mathrm{Ar}$ reaction between 4-chloro-2-trichloromethylquinazoline 1and benzyl alcohol. To facilitate the $S_{N} A r$ reaction between 1 and poorly nucleophilic species like amino- 
substituted heterocycles, our group recently reported a synthetic procedure using $N-N$ dimethylaminopyridine (DMAP) as an efficient catalyst, involving the formation of a highly reactive quinazoline intermediate which we isolated and characterized by $\mathrm{X}$-ray diffraction [25]. Then, benefiting from our experience in the use of microwave irradiation for introducing substituents at position 4 of the quinazoline ring [26], and considering that a successful microwave-assisted $S_{N} A r$ reaction between a 4-chloroquinazoline derivative and ethylene glycol had been reported [27], we focused on a $S_{N} A r$ reaction protocol combining a DMAP catalysis with microwave irradiation.For that purpose, we first prepared substrate 1 (Figure 1), from commercial 2-methylquinazolin-4(3H)-one, according to a previously reported microwave synthesis using $\mathrm{PCl}_{5}$ in $\mathrm{POCl}_{3}$ [28].

Figure 1. Microwave-assisted synthesis of substrate 1 from commercial 2-methylquinazolin$4(3 H)$-one.

Then, the DMAP-catalyzed $S_{N} A r$ reaction between 1 and benzyl alcohol was studied (Figure 2) by varying the solvent nature, the amount of catalyst, the amount of benzyl alcohol, the reaction temperature, the microwave irradiation mode and the reaction time. The experimental results are presented in Table 2.

Figure 2. Studied reaction: preparation of 4-benzyloxy-2-trichloromethylquinazoline 2 from substrate 1

When using DMAP in the presence of toluene, the reaction yield reached 23\%, higher than with DMSO, DMF, or THF. Then, by increasing the amount of DMAP to 0.2 equiv., the reaction yield was improved to $42 \%$. Finally, via a $1 \mathrm{~h}$ monomode microwave irradiation at $130^{\circ} \mathrm{C}$, the use of 0.3 equiv. of DMAP, allowed a decrease in the amount of alcohol $(1,2$ equiv.) needed and a53\% reaction yield was reached. From that point, decreasing reaction temperature, reaction time, or using a multimode microwave irradiation did not provide satisfying reaction yields. 
Table 2. Studied parameters for the DMAP-catalyzed reaction of 1 with benzyl alcohol

\begin{tabular}{|c|c|c|c|c|c|}
\hline Entry & $\begin{array}{l}\text { DMAP } \\
(\mathrm{mol} \%)\end{array}$ & Reaction conditions $^{\mathrm{a}}$ & $\begin{array}{l}\text { Alcohol } \\
\text { equiv. }\end{array}$ & Solvent & $\begin{array}{l}\text { Yield } \\
(\%)\end{array}$ \\
\hline 1 & 10 & $1 \mathrm{~h}, 130^{\circ} \mathrm{C} /$ monomode MW & 2 & toluene & 23 \\
\hline 2 & 10 & $1 \mathrm{~h}, 150{ }^{\circ} \mathrm{C} /$ monomode $\mathrm{MW}$ & 2 & DMSO & $5^{\mathrm{b}}$ \\
\hline 3 & 10 & $1 \mathrm{~h}, 150^{\circ} \mathrm{C} /$ monomode $\mathrm{MW}$ & 2 & DMF & $3^{\mathrm{b}}$ \\
\hline 4 & 10 & $1 \mathrm{~h}, 80^{\circ} \mathrm{C} /$ monomode $\mathrm{MW}$ & 2 & THF & Traces $^{\mathrm{c}}$ \\
\hline 5 & 20 & $1 \mathrm{~h}, 130^{\circ} \mathrm{C} /$ monomode MW & 2 & toluene & 42 \\
\hline 6 & 30 & $0.5 \mathrm{~h}, 130^{\circ} \mathrm{C} /$ monomode $\mathrm{MW}$ & 2 & toluene & 27 \\
\hline 7 & 40 & $1 \mathrm{~h}, 130^{\circ} \mathrm{C} /$ monomode $\mathrm{MW}$ & 2 & toluene & 51 \\
\hline 8 & 30 & $1 \mathrm{~h}, 130{ }^{\circ} \mathrm{C} /$ monomode $\mathrm{MW}$ & 1.2 & toluene & 53 \\
\hline 9 & 30 & $1 \mathrm{~h}, 130^{\circ} \mathrm{C} /$ monomode MW & 2 & toluene & 53 \\
\hline 10 & 30 & $2 \mathrm{~h}, 110^{\circ} \mathrm{C} /$ monomode $\mathrm{MW}$ & 2 & toluene & $39^{\mathrm{b}}$ \\
\hline 11 & 40 & $0.5 \mathrm{~h}, 150^{\circ} \mathrm{C} /$ monomode $\mathrm{MW}$ & 2 & toluene & $18^{\mathrm{b}}$ \\
\hline 12 & 40 & $2 \mathrm{~h}, 800 \mathrm{~W}, 110^{\circ} \mathrm{C} /$ multimode $\mathrm{MW}$ & 2 & toluene & 27 \\
\hline 13 & - & $\mathrm{NaH} 95 \%$ (2 equiv.), RT, 24 h [19] & 1 & DMSO & 11 \\
\hline
\end{tabular}

\footnotetext{
${ }^{a}$ Themonomode microwave oven used was a Biotage Initiator. The multimode microwave oven used was a Milestone SynthLab Station ${ }^{\mathrm{b}}$ Formation of resins

${ }^{\mathrm{c}}$ Detected by LC/MS
}

For comparison purposes, the same reaction was carried out without DMAP and then conducted with other tertiary amine derivatives (DBU, DABCO and TEA). As shown in Table 3, under these reaction conditions, the presence ofDMAP appears mandatory for the reaction to proceed. Moreover, among tertiary amines, only DMAP allowed the synthesis of 2, considering that substrate $\mathbf{1}$ was found unconsumed after reaction in the conditions of entries 3 and 4 (only 5\% conversion with TEA).

Table 3. Tertiary amine catalysts evaluated for the synthesis of 2

\begin{tabular}{ccc}
\hline Entry & Catalyst* $^{*}$ & Yield (\%) \\
\hline 1 & - & 0 \\
2 & DMAP & 53 \\
3 & DBU & 0 \\
4 & DABCO & 0 \\
\hline
\end{tabular}




\begin{tabular}{lll}
\hline 5 & TEA & 5 \\
\hline & $* 0.3$ equiv.
\end{tabular}

According to this general procedure, summarized in Figure 3, the $S_{N} A r$ reaction of 1 with aliphatic alcohols (mainly benzyl alcohols) was then extended to the synthesis of a series of 18 derivatives (molecules 2-19). As presented in Table 4, the reaction yields varied from moderate $(35 \%)$ to excellent $97 \%$. In addition, the same protocol was applied to the synthesis of a series of 13 derivatives bearing a phenoxy moiety at position 4 of the quinazoline ring (molecules 20-32). These molecules were obtained in 38-99\% yields. Finally, 4 more derivatives were prepared, bearing a heteroaryloxy moiety at position 4 of the quinazoline ring (molecules 33-36), in 55-98\% yields. Thus, this DMAP-catalyzed operating procedure appeared as generally applicable to aliphatic alcohols, phenols and hydroxyheteroaryl reagents.

Figure 3. Optimal conditions for preparing derivatives belonging to the 4-benzyloxy-2trichloromethylquinazoline series from substrate 1

Table 4. Reaction yields and structures of synthesized compounds 2-36

\begin{tabular}{cccccc}
\hline R- & $\mathrm{N}^{\circ}$ & Yield (\%) & R- & $\mathrm{N}^{\circ}$ & Yield (\%) \\
\hline $\mathbf{2}$ & 53 & $\mathbf{2 0}$ & 99 \\
$\mathbf{3}$ & $\mathbf{7 2}$ & $\mathbf{2 1}$ & 99 \\
& $\mathbf{4}$ & 65 & $\mathbf{2 2}$ & 99 \\
$\mathbf{5}$ & 97 & $\mathbf{2 3}$ & 38 \\
& $\mathbf{6}$ & 96 & & \\
& & $\mathbf{2 4}$ & 95 \\
\hline
\end{tabular}




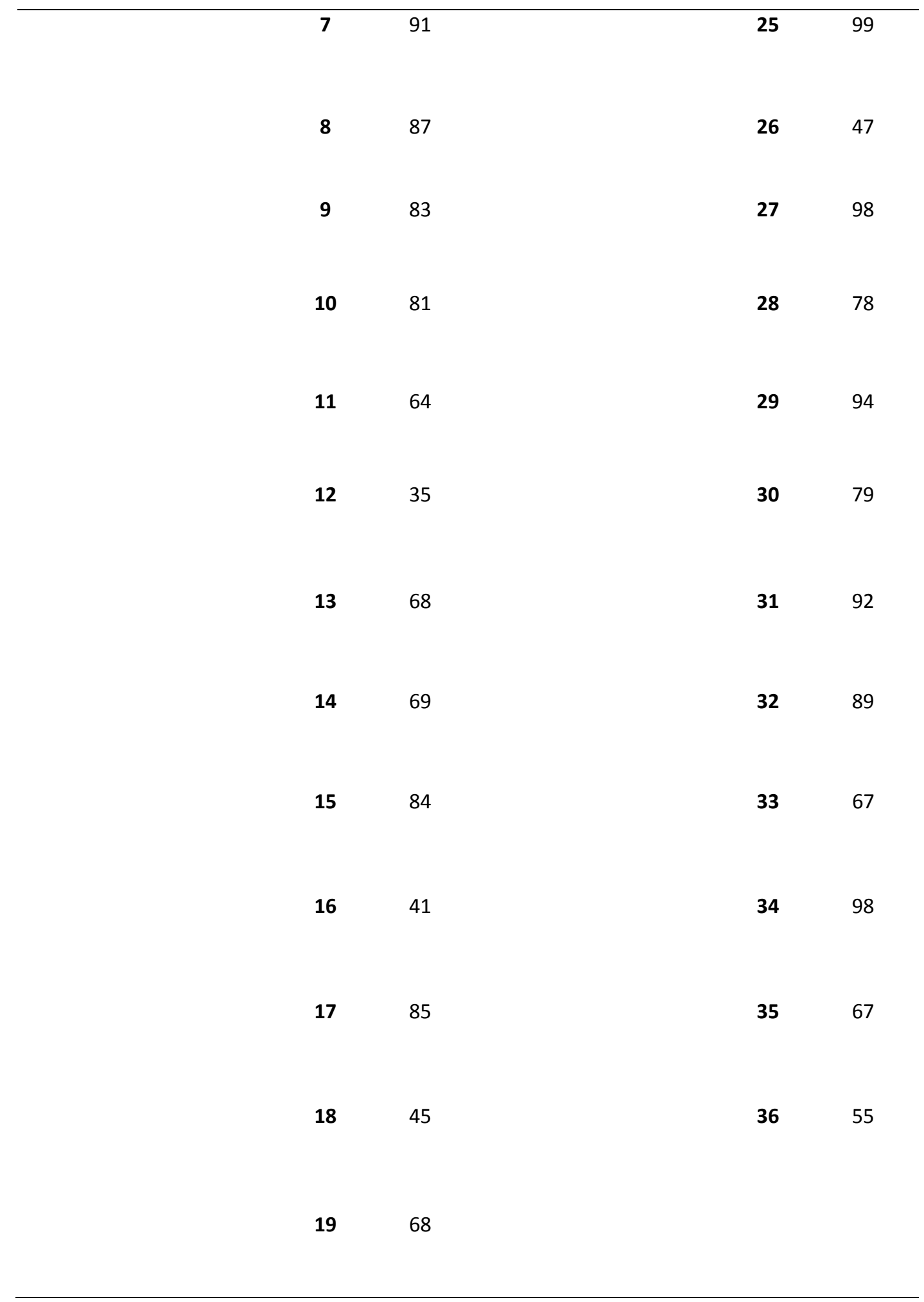

Among the 35 synthesized molecules, 2 were previously reported. Thus, compound 2 was mentioned in a 2001 publication [29] but no experimental data is available about it up to day. Concerning quinazoline27, it is to note that it had previously been synthesized, according to another protocol [19], in 25\% yield. As shown in Figure 4, from the same substrate, the presently described DMAP protocol led to the formation of product 27 in $98 \%$ yield and only 
$1 \mathrm{~h}$, indicating its high efficiency for preparing most of 4-phenoxyquinazolines (apart for molecules 23 and 26).

Figure 4. Comparison of synthetic routes A and B [19] for the synthesis of compound 27

\subsection{Biological evaluation}

All synthesized molecules were then evaluated in vitro on the multi-resistant $\mathrm{K} 1 P$. falciparum strain, by determining their $50 \%$ inhibitory concentration $\left(\mathrm{IC}_{50}\right)$, and compared with 2 antimalarial drug-compounds: chloroquine and doxycycline. In parallel, these molecules were assessed in vitro on the HepG2 human hepatocyte cell line, by determining their $50 \%$ cytotoxic concentrations $\left(\mathrm{CC}_{50}\right)$ and comparing them to the one of doxorubicin, used as a cytotoxic reference drug-compound, in order to calculate their respective selectivity index $\left(\mathrm{SI}=\mathrm{CC}_{50} / \mathrm{IC}_{50}\right)$. The results are presented in Table 5 and highlight 5 hit-molecules $(\mathbf{2}$, 6, 16, 20, 26), presenting both $\mathrm{IC}_{50}$ values $<2 \mu \mathrm{M}$ and $\mathrm{CC}_{50}$ values $>25 \mu \mathrm{M}$, reaching selectivity indexes ranging between 17 and 27.

Table 5.In vitro antiplasmodial and cytotoxicity evaluation of the 4-benzyloxy-2-trichloromethylquinazolines series

\begin{tabular}{|c|c|c|c|c|}
\hline Molecule & R- & $\begin{array}{c}\text { HepG2 } \\
\mathrm{CC}_{50}(\mu \mathrm{M})\end{array}$ & $\begin{array}{c}\text { PfK1 IC } \\
(\mu \mathrm{M})\end{array}$ & $\mathbf{S I}^{\mathrm{e}}$ \\
\hline 2 & & 43.8 & 2.0 & 21.9 \\
\hline 3 & & 50.9 & 4.0 & 12.7 \\
\hline 4 & & 90.3 & $>10^{\mathrm{d}}$ & $<9$ \\
\hline
\end{tabular}




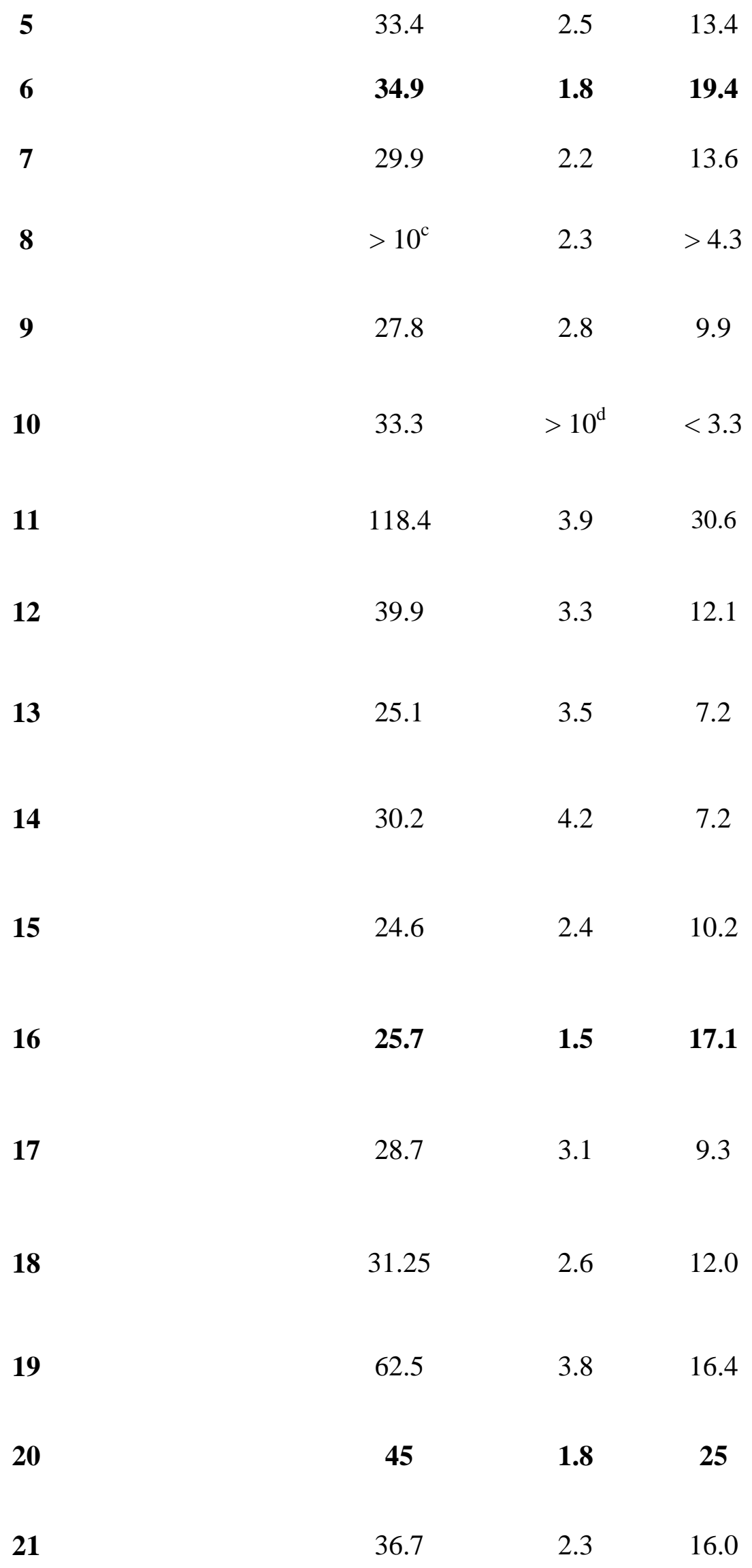


Doxorubicine $^{\mathrm{a}}$

59.0

$>10^{\mathrm{d}}$

$<5.9$

\section{Chloroquine $^{b}$}

0.2

\section{Doxycycline $^{\text {b }}$}

0.6 50

20.0

6.0 3.3

${ }^{a}$ Doxorubicine was used as a cytotoxic reference-drug;

${ }^{\mathrm{b}} \mathrm{Chloroquinine}$ and doxycycline were used as antimalarial reference-drugs;

${ }^{\mathrm{c}} \mathrm{CC}_{50}$ could not be determined because of a lack of solubility of the tested molecule in the culture medium; 
${ }^{\mathrm{d}} \mathrm{IC}_{50}$ was not reached at the highest tested concentration $(10 \mu \mathrm{M})$.

${ }^{\text {e}}$ Selectivity index (SI) was calculated according to the formula: $\mathrm{SI}=\mathrm{CC}_{50} / \mathrm{IC}_{50}$;

In bold: hit-compounds $\left(\mathrm{IC}_{50} \leq 2 \mu \mathrm{M}, \mathrm{CC}_{50} \geq 25 \mu \mathrm{M}\right.$ and $\left.\mathrm{SI} \geq 17\right)$

\subsection{Structure-activity relationships}

Apart compounds $\mathbf{8}$ and $\mathbf{2 3}$, all tested molecules presented satisfying solubilities in the biological media as the determination of their in vitro activities was not hindered by any precipitation. In the benzyloxy series, no cytotoxicity was identified whereas, in the phenoxy series, compounds 25, 27 and 31, presenting a substituent at the meta position of the benzene ring, displayed low $\mathrm{CC}_{50}$ values $(0.5-5 \mu \mathrm{M})$. In the heteroaryloxy series, compound 34 also appeared slightly cytotoxic $\left(\mathrm{CC}_{50}=5 \mu \mathrm{M}\right)$, because of the presence of two bromine atoms.

Concerning antiplasmodial activity, the molecules belonging to the heteroaryloxy series were not active. In the benzyloxy series, compounds 2, $\mathbf{6}$ and $\mathbf{1 6}$ appeared as hit-molecules with $\mathrm{IC}_{50}$ values ranging from 1.5 to $2 \mu \mathrm{M}$ and selectivity indexes being around 20 , in comparison with the values noted for reference drugs.Compound 2 , bearing a benzyloxy group at position 4 of the quinazoline ring, was found more active and selective than its phenyloxy analog $\left(\mathrm{CI}_{50}=3,1 \mu \mathrm{M} / \mathrm{SI}=16\right)$ [19]. In most cases (apart for nitrated molecule $\left.\mathbf{6}\right)$, the substitution of the benzene ring of the benzyloxy group was globally deleterious toward antiplasmodial activity, nevertheless, considering compound 16, it can be noted that the introduction of a substituent on the benzylic carbon, for example via a nitrile function, is compatible with maintaining biological activity.

In the phenoxy series, compounds $\mathbf{2 0}$ and $\mathbf{2 6}$ were identified as hit-molecules, displaying $\mathrm{IC}_{50}$ values of 1.8 and $1.5 \mu \mathrm{M}$ and selectivity indexes above 25 . When comparing para-brominated molecule 20 to its chlorinated analog (Table 1), in accordance with the SAR conclusions that we previously reported [19], it can be concluded that the substitution of the benzene ring by a chlorine or bromine atom is favorable toward the antiplasmodial activity if located at the para position of the benzene ring, chlorine remaining slightly more favorable. Indeed, we also identified that the introduction of an acetyl substituent at the ortho position of the benzene ring (compound 26) allowed antiplasmodial activity. Thisselective activity is decreased when changing the substitution position or when changing the acetyl group into a formyl analog (compound 28). To validate the key role played by the 2-trichloromethylquinazoline scaffold of hit-molecule 26, its dehalogenated analog 37 was prepared in two steps (Figure 5).

Figure 5. Two step synthesis of molecule 37 
As presented in Figure 6, the antiplasmodial activity of compound $\mathbf{2 6}$ depends from the presence of a trichloromethyl group at position 2 of the quinazoline ring, in accordance with the results that we previously reported. Thus, compounds $\mathbf{2}$ (in the original benzyloxy series) and 26 (first active $o$-substituted phenoxy derivative) appear as novel hit-molecules and extend the available SAR data in the 2-trichloromethylquinazoline series.

Figure 6. Comparison of the in vitro antiplasmodial activities of molecules $\mathbf{2 6}$ and $\mathbf{3 7}$

\section{Conclusion}

Five antiplasmodial hit-molecules were identified from an in vitro screening of 35 new 2trichloromethylquinazoline derivatives bearing an oxygen-containing moiety at position 4 of the quinazoline ring. Structure-activity relationships showed that the derivatives presenting a benzyloxy, a $p$-bromophenoxy or an $o$-acetylphenoxy moiety were the most active and selective ones. The global series was prepared from 4-chloro-2-trichloromethylquinazoline via a DMAP catalyzed $S_{N} A r$ reaction with benzylic alcohols, phenols or hydroxyheterocycles, under microwave irradiation. This operating procedure appears as an efficient, simple, fast and cheap operating protocol for introducing a large diversity of alkoxy, phenoxy or heteroaryloxy substituents at position 4 of 4-chloroquinazolines, substrates of wide pharmaceutical interest. 


\section{Experimental}

\subsection{Chemistry}

\subsubsection{General}

Melting points were determined on a Köfler melting point apparatus and are uncorrected. Elemental analyses were carried out at the Spectropole, Faculté des Sciences de Saint-Jêrome (Marseille) with a ThermoFinnigan EA1112 analyzer and a BruckerNonius diffractometer. NMR spectra were recorded on a Bruker ARX 200 spectrometer at the Faculté de Pharmacie of Marseille (200 MHz ${ }^{1} \mathrm{H}$ NMR: reference $\mathrm{CHCl}_{3} \delta=7.26$, and $50 \mathrm{MHz}{ }^{13} \mathrm{C}$ : reference $\mathrm{CHCl}_{3} \delta=76.9$ ). The following adsorbent was used for column chromatography: silica gel 60 (Merck, particle size 0.063-0.200 mm, 70-230 mesh ASTM). TLC was performed on $5 \mathrm{~cm}$ $\mathrm{x} 10 \mathrm{~cm}$ aluminum plates coated with silica gel 60F-254 (Merck) in an appropriate eluent. Visualization was performed with ultraviolet light (234 nm). Purity of synthesized compounds was checked by LC/MS analyses, which were realized at the Faculté de Pharmacie of Marseille with a Thermo Scientific Accela High Speed LC System ${ }^{\circledR}$ coupled using a single quadrupole mass spectrometer Thermo MSQ Plus®. The RP-HPLC column is a ThermoHypersil Gold ${ }^{\circledR} 50 \times 2.1 \mathrm{~mm}$ (C18 bounded), with particles of a diameter of $1.9 \mathrm{~mm}$. The volume of sample injected on the column is $1 \mu \mathrm{L}$. Chromatographic analysis, total duration of $8 \mathrm{~min}$, is on the gradient of the following solvents: $\mathrm{t}=0 \mathrm{~min}$, methanol/water 50:50; $0<\mathrm{t}<4 \mathrm{~min}$, linear increase in the proportion of water to a methanol/water ratio of 95:5; $4<\mathrm{t}<6 \mathrm{~min}$, methanol/water $95: 5 ; 6<\mathrm{t}<7 \mathrm{~min}$, linear decrease in the proportion of water to return to a methanol/water ratio of $50: 50 ; 6<t<7 \mathrm{~min}$, methanol/water 50:50. The water used was buffered with ammonium acetate $5 \mathrm{mM}$.The flow rate of the mobile phase was 0.3 $\mathrm{mL} / \mathrm{min}$. The retention times $\left(t_{\mathrm{R}}\right)$ of the molecules analyzed are indicated in min. The microwave reactions were performed using multimode reactors: ETHOS Synth Lab station and MicroSYNTH ${ }^{\circledR}$ Lab terminal 1024 (Ethos start, Milestone Inc.); or monomode reactors: Biotage Initiator® classic in sealed vials.

2-Methylquinazolin-4(3H)-one was purchased from Sigma Aldrich.

\subsubsection{4-Chloro-2-trichloromethylquinazoline(1)}

4-Chloro-2-trichloromethylquinazoline $\mathbf{1}$ was prepared as described in the literature [28].Yield 82\%. White solid. Mp $127{ }^{\circ} \mathrm{C}$ (lit. $\left.127{ }^{\circ} \mathrm{C}\right) .{ }^{61} \mathrm{H} \mathrm{NMR}\left(\mathrm{CDCl}_{3}, 200 \mathrm{MHz}\right) \delta=7.82$ - 
$7.90(\mathrm{~m}, 1 \mathrm{H}), 8.03-8.12(\mathrm{~m}, 1 \mathrm{H}), 8.20-8.24(\mathrm{~m}, 1 \mathrm{H}), 8.33-8.38(\mathrm{~m}, 1 \mathrm{H}) . \mathrm{C}_{9} \mathrm{H}_{4} \mathrm{Cl}_{4} \mathrm{~N}_{2}, \mathrm{MW}$ : $282.18 \mathrm{~g} / \mathrm{mol}$.

\subsubsection{General procedure for the preparation of compounds 2 to 34}

A mixture of 4-chloro-2-trichloromethylquinazoline 1 (0.2 g, $0.71 \mathrm{mmol})$, DMAP (26 mg, $0.21 \mathrm{mmol}, 0.3$ equiv) and adequate alcohol derivative $(0.85 \mathrm{mmol}, 1.2$ equiv) in toluene ( 3 $\mathrm{mL})$ was introduced in miniaturized sealed reactor $(5 \mathrm{~mL})$. The reaction mixture was irradiated in a monomode microwave oven, for $1 \mathrm{~h}$ at $130{ }^{\circ} \mathrm{C}$. After removal of the toluene under reduced pressure, the residue was purified by silica gel column chromatography and recrystallized from appropriate solvent.

\subsubsection{4-(Benzyloxy)-2-(trichloromethyl)quinazoline (2)}

Yield 53\%. Beige powder. Mp $115{ }^{\circ} \mathrm{C}$, (isopropanol). ${ }^{1} \mathrm{H}$ NMR $\left(200 \mathrm{MHz}, \mathrm{CDCl}_{3}\right) \delta=8.23$ $(\mathrm{dd}, J=1 \mathrm{~Hz} ; 8 \mathrm{~Hz}, 1 \mathrm{H}), 8.07$ (d, $J=8 \mathrm{~Hz}, 1 \mathrm{H}), 7.88$ (td, $J=1 \mathrm{~Hz} ; 5 \mathrm{~Hz}, 1 \mathrm{H}), 7.66-7.58$ (m, $3 \mathrm{H}), 7.45-7.37(\mathrm{~m}, 3 \mathrm{H}), 5.74(\mathrm{~s}, 2 \mathrm{H}) .{ }^{13} \mathrm{C} \mathrm{NMR}\left(50 \mathrm{MHz}, \mathrm{CDCl}_{3}\right) \delta=167.7,160.1,150.4$, 135.6, 134.4, 129.0, 128.7, 128.6, 123.7, 115.5, 97.3, 69.4. LC-MS (ESI+) $t_{R} 5.38 \mathrm{~min}, \mathrm{~m} / \mathrm{z}$ $[\mathrm{M}+\mathrm{H}]^{+}$351.79/354.40/355.98. MW: $353.63 \mathrm{~g} / \mathrm{mol}$. Anal. Calcd for $\mathrm{C}_{16} \mathrm{H}_{11} \mathrm{Cl}_{3} \mathrm{~N}_{2} \mathrm{O}: \mathrm{C}, 54.34$; H, 3.14; N, 7.92. Found: C, 54.45; H, 3.11; N, 7.98.

\subsubsection{4-(4-Methylbenzyloxy)-2-(trichloromethyl)quinazoline (3)}

Yield $72 \%$. Pale beige powder. Mp $130^{\circ} \mathrm{C}$, (isopropanol). ${ }^{1} \mathrm{H}$ NMR $\left(200 \mathrm{MHz}, \mathrm{CDCl}_{3}\right) \delta=$ $8.23(\mathrm{~d}, J=8 \mathrm{~Hz}, 1 \mathrm{H}), 8.07$ (d, $J=8 \mathrm{~Hz}, 1 \mathrm{H}), 7.90$ (t, $J=8 \mathrm{~Hz}, 1 \mathrm{H}), 7.67-7.53(\mathrm{~m}, 1 \mathrm{H}), 7.53-$ $7.49(\mathrm{~d}, J=8 \mathrm{~Hz}, 3 \mathrm{H}), 7.23-7.19(\mathrm{~d}, J=8 \mathrm{~Hz}, 1 \mathrm{H}), 5.70(\mathrm{~s}, 2 \mathrm{H}), 2.37$ (s, 3H). ${ }^{13} \mathrm{C}$ NMR $(50$ $\left.\mathrm{MHz}, \mathrm{CDCl}_{3}\right) \delta=167.6,160.1,150.4,138.5,134.4,132.6,129.2,129.3,128.7,128.6,123.7$, 115.6, 97.6, 69.5, 27.3. LC-MS (ESI+) $t_{R} 5.68 \mathrm{~min}, \mathrm{~m} / \mathrm{z}[\mathrm{M}+\mathrm{H}]^{+} 367.11 / 368.98 / 371.30$. MW: $367.66 \mathrm{~g} / \mathrm{mol}$. Anal. Calcd for $\mathrm{C}_{17} \mathrm{H}_{13} \mathrm{Cl}_{3} \mathrm{~N}_{2} \mathrm{O}$ : C, 55.54; H, 3.56; N, 7.62. Found: C, 55.39; H, $3.53 ; \mathrm{N}, 7.51$.

\subsubsection{4-(4-Methoxybenzyloxy)-2-(trichloromethyl)quinazoline (4)}

Yield 65\%. Pale beige powder. $\mathrm{Mp} 86{ }^{\circ} \mathrm{C}$, (isopropanol). ${ }^{1} \mathrm{H} \mathrm{NMR}\left(200 \mathrm{MHz}, \mathrm{CDCl}_{3}\right) \delta=8.21$ $(\mathrm{d}, J=8 \mathrm{~Hz}, 1 \mathrm{H}), 8.06(\mathrm{~d}, J=8 \mathrm{~Hz}, 1 \mathrm{H}), 7.90(\mathrm{t}, J=8 \mathrm{~Hz}, 1 \mathrm{H}), 7.67-7.54(\mathrm{~m}, 3 \mathrm{H}), 6.94-6.90$ $(\mathrm{d}, J=8 \mathrm{~Hz}, 2 \mathrm{H}), 5.68(\mathrm{~s}, 2 \mathrm{H}), 3.82(\mathrm{~s}, 3 \mathrm{H}) .{ }^{13} \mathrm{C} \mathrm{NMR}\left(50 \mathrm{MHz}, \mathrm{CDCl}_{3}\right) \delta=167.6,159.9$, 150.4, 134.4, 131.0, 128.7, 128.6, 127.7, 126.9, 123.7, 115.6, 114.1, 114.0, 97.2, 69.4, 55.3. 
LC-MS (ESI+) $t_{R} 5.33 \mathrm{~min}, \mathrm{~m} / \mathrm{z}[\mathrm{M}+\mathrm{H}]^{+}$382.94/384.77/386.88. MW: $383.66 \mathrm{~g} / \mathrm{mol}$. Anal. Calcd for $\mathrm{C}_{17} \mathrm{H}_{13} \mathrm{Cl}_{3} \mathrm{~N}_{2} \mathrm{O}_{2}$ : C, 53.22; H, 3.42; N, 7.30. Found: C, 53.13; H, 3.39; N, 7.51.

\subsubsection{4-(4-Chlorobenzyloxy)-2-(trichloromethyl)quinazoline (5)}

Yield 97\%. Beige powder. Mp $95{ }^{\circ} \mathrm{C}$, (isopropanol). ${ }^{1} \mathrm{H}$ NMR $\left(200 \mathrm{MHz}, \mathrm{CDCl}_{3}\right) \delta=8.22(\mathrm{~d}$, $J=8 \mathrm{~Hz}, 1 \mathrm{H}), 8.08(\mathrm{~d}, J=8 \mathrm{~Hz}, 1 \mathrm{H}), 7.90(\mathrm{td}, J=2 \mathrm{~Hz} ; 7 \mathrm{~Hz}, 1 \mathrm{H}), 7.69-7.61(\mathrm{~m}, 1 \mathrm{H}), 7.57-$ $7.53(\mathrm{~d}, J=8 \mathrm{~Hz}, 2 \mathrm{H}), 7.39-7.34(\mathrm{~d}, J=8 \mathrm{~Hz}, 2 \mathrm{H}), 5.69(\mathrm{~s}, 2 \mathrm{H}) .{ }^{13} \mathrm{C} \mathrm{NMR}\left(50 \mathrm{MHz}, \mathrm{CDCl}_{3}\right)$ $\delta=167.4,160.0,150.4,134.5,134.1,130.5,128.8,128.7,123.6,115.5,97.1,68.7$. LC-MS $(\mathrm{ESI}+) t_{R} 5.59 \mathrm{~min}, \mathrm{~m} / \mathrm{z}[\mathrm{M}+\mathrm{H}]^{+} 386.61 / 388.96 / 390.91$. MW: $388.08 \mathrm{~g} / \mathrm{mol}$. Anal. Calcd for $\mathrm{C}_{16} \mathrm{H}_{10} \mathrm{Cl}_{4} \mathrm{~N}_{2} \mathrm{O}: \mathrm{C}, 49.52 ; \mathrm{H}, 2.60 ; \mathrm{N}, 7.22$. Found: C, 49.70; H, 2.58; N, 7.14.

\subsubsection{4-(4-Nitrobenzyloxy)-2-(trichloromethyl)quinazoline (6)}

Yield 96\%. Yellow powder. Mp $199{ }^{\circ} \mathrm{C}$, (isopropanol). ${ }^{1} \mathrm{H} \mathrm{NMR}\left(200 \mathrm{MHz}, \mathrm{CDCl}_{3}\right) \delta=8.34$ $8.30(\mathrm{~m}, 3 \mathrm{H}), 8.18-8.15(\mathrm{~m}, 1 \mathrm{H}), 8.04-7.98(\mathrm{~m}, 1 \mathrm{H}), 7.85-7.74(\mathrm{~m}, 3 \mathrm{H}), 5.88(\mathrm{~s}, 2 \mathrm{H}) .{ }^{13} \mathrm{C}$ NMR $\left(50 \mathrm{MHz}, \mathrm{CDCl}_{3}\right) \delta=167.1,159.8,150.5,147.9,142.8,134.8,129.3,129.1,129.0$, 123.9, 123.4, 115.3, 97.0, 68.0. LC-MS (ESI+) $t_{R} 5.06 \quad \mathrm{~min}, \quad \mathrm{~m} / \mathrm{z} \quad[\mathrm{M}+\mathrm{H}]^{+}$ 397.93/399.91/402.06. MW: $398.63 \mathrm{~g} / \mathrm{mol}$. Anal. Calcd for $\mathrm{C}_{16} \mathrm{H}_{10} \mathrm{Cl}_{3} \mathrm{~N}_{3} \mathrm{O}_{3}: \mathrm{C}, 48.21 ; \mathrm{H}$, 2.53 ; N, 10.54. Found: C, 48.33; H, 2.55; N, 10.41 .

\subsubsection{4-(3-Nitrobenzyloxy)-2-(trichloromethyl)quinazoline (7)}

Yield $91 \%$. Yellow powder. Mp $147{ }^{\circ} \mathrm{C}$, (isopropanol). ${ }^{1} \mathrm{H}$ NMR $\left(200 \mathrm{MHz}, \mathrm{CDCl}_{3}\right) \delta=8.27$ $8.24(\mathrm{~m}, 1 \mathrm{H}), 8.19-8.14(\mathrm{~m}, 2 \mathrm{H}), 7.88-7.81(\mathrm{~m}, 3 \mathrm{H}), 7.69-7.54(\mathrm{~m}, 2 \mathrm{H}), 5.95(\mathrm{~s}, 2 \mathrm{H}) .{ }^{13} \mathrm{C}$ NMR $\left(50 \mathrm{MHz}, \mathrm{CDCl}_{3}\right) \delta=167.1,159.7,150.3,148.0,134.9,133.5,131.7,130.1,129.3$, 128.9, 128.8, 124.9, 123.2, 115.1, 97.2, 67.8. LC-MS (ESI+) $t_{R} 4.95 \mathrm{~min}, \mathrm{~m} / \mathrm{z}[\mathrm{M}+\mathrm{H}]^{+}$ 397.94/399.89/401.96. MW: $398.63 \mathrm{~g} / \mathrm{mol}$. Anal. Calcd for $\mathrm{C}_{16} \mathrm{H}_{10} \mathrm{Cl}_{3} \mathrm{~N}_{3} \mathrm{O}_{3}: \mathrm{C}, 48.21 ; \mathrm{H}$, $2.53 ; \mathrm{N}, 10.54$. Found: $\mathrm{C}, 48.03 ; \mathrm{H}, 2.56 ; \mathrm{N}, 10.66$.

\subsubsection{4-(2-Nitrobenzyloxy)-2-(trichloromethyl)quinazoline (8)}

Yield 87\%. Yellow powder. Mp $141{ }^{\circ} \mathrm{C}$, (isopropanol). ${ }^{1} \mathrm{H}$ NMR $\left(200 \mathrm{MHz}, \mathrm{CDCl}_{3}\right) \delta=8.28$ $8.23(\mathrm{~m}, 1 \mathrm{H}), 8.14-8.07(\mathrm{~m}, 2 \mathrm{H}), 7.94(\mathrm{td}, J=2 \mathrm{~Hz} ; 7 \mathrm{~Hz}, 1 \mathrm{H}), 1 \mathrm{H}), 7.82-7.48(\mathrm{~m}, 4 \mathrm{H}), 6.13$ (s, 2H). ${ }^{13} \mathrm{C}$ NMR $\left(50 \mathrm{MHz}, \mathrm{CDCl}_{3}\right) \delta=167.0,159.9,150.5,148.2,134.7,133.6,131.7$, 130.3, 129.2, 129.0, 128.9, 125.1, 123.5, 115.3, 96.9, 66.1. LC-MS (ESI+) $t_{R} 4.82 \mathrm{~min}, \mathrm{~m} / \mathrm{z}$ 
$[\mathrm{M}+\mathrm{H}]^{+}$397.91/400.02/401.86. MW: $398.63 \mathrm{~g} / \mathrm{mol}$. Anal. Calcd for $\mathrm{C}_{16} \mathrm{H}_{10} \mathrm{Cl}_{3} \mathrm{~N}_{3} \mathrm{O}_{3}: \mathrm{C}$, 48.21; H, 2.53; N, 10.54. Found: C, 48.31; H, 2.50; N, 10.39.

\subsubsection{2-(Trichloromethyl)-4-(4-(trifluoromethyl)benzyloxy)quinazoline (9)}

Yield $83 \%$. Pale yellow powder. Mp $130{ }^{\circ} \mathrm{C}$, (isopropanol). ${ }^{1} \mathrm{H}$ NMR $\left(200 \mathrm{MHz}, \mathrm{CDCl}_{3}\right) \delta=$ 8.27-8.23 (m, 1H), 8.18-8.12 (m, 1H), 8.04-7.98 (m, 1H), 7.97-7.89 (m, 1H),7.75-7.64 (m, $4 \mathrm{H}), 5.79(\mathrm{~s}, 2 \mathrm{H}) .{ }^{13} \mathrm{C} \mathrm{NMR}\left(50 \mathrm{MHz}, \mathrm{CDCl}_{3}\right) \delta=167.3,160.0,150.4,139.6,134.7,129.0$, 128.9, 126.7, 125.6, 125.5, 123.5, 121.3, 115.4, 97.1, 68.5. LC-MS (ESI+) $t_{R} 5.52 \mathrm{~min}, \mathrm{~m} / \mathrm{z}$ $[\mathrm{M}+\mathrm{H}]^{+}$420.88/422.92/424.93. MW: $421.63 \mathrm{~g} / \mathrm{mol}$. Anal. Calcd for $\mathrm{C}_{17} \mathrm{H}_{10} \mathrm{Cl}_{3} \mathrm{~F}_{3} \mathrm{~N}_{2} \mathrm{O}: \mathrm{C}$, 48.43; H, 2.39; N, 6.64. Found: C, 48.59; H, 2.41; N, 6.58.

\subsubsection{4-(4-Isopropylbenzyloxy)-2-(trichloromethyl)quinazoline (10)}

Yield $81 \%$. Pale beige powder. Mp121 ${ }^{\circ} \mathrm{C}$, (isopropanol). ${ }^{1} \mathrm{H}$ NMR $\left(200 \mathrm{MHz}, \mathrm{CDCl}_{3}\right) \delta=$ $8.23(\mathrm{~d}, J=8 \mathrm{~Hz}, 1 \mathrm{H}), 8.07$ (d, $J=8 \mathrm{~Hz}, 1 \mathrm{H}), 7.93-7.85$ (m, 1H), 7.67-7.53(m, 3H), 7.28 (d, $J$ $=8 \mathrm{~Hz}, 2 \mathrm{H}), 5.72(\mathrm{~s}, 2 \mathrm{H}), 2.94(\mathrm{sept}, J=7 \mathrm{~Hz}, 1 \mathrm{H}), 1.27(\mathrm{~d}, J=7 \mathrm{~Hz}, 6 \mathrm{H}) \cdot{ }^{13} \mathrm{C} \mathrm{NMR}(50$ $\left.\mathrm{MHz}, \mathrm{CDCl}_{3}\right) \delta=167.6,160.2,150.4,149.5,134.4,133.0,129.3,128.7,128.6,126.7,123.8$, 115.6, 97.3, 68.5, 34.0, 24.0. LC-MS (ESI+) $t_{R} 5.73 \mathrm{~min}, \mathrm{~m} / \mathrm{z}[\mathrm{M}+\mathrm{H}]^{+} 395.10 / 396.96 / 399.02$. MW: $395.71 \mathrm{~g} / \mathrm{mol}$. Anal. Calcd for $\mathrm{C}_{19} \mathrm{H}_{17} \mathrm{Cl}_{3} \mathrm{~N}_{2} \mathrm{O}: \mathrm{C}, 57.67 ; \mathrm{H}, 4.33 ; \mathrm{N}, 7.08$. Found: C, $57.76 ; \mathrm{H}, 4.35 ; \mathrm{N}, 7.21$.

\subsubsection{4-(3,4-Dimethoxybenzyloxy)-2-(trichloromethyl)quinazoline (11)}

Yield 64\%. Beige powder. Mp127 ${ }^{\circ} \mathrm{C}$, (isopropanol). ${ }^{1} \mathrm{H}$ NMR $\left(200 \mathrm{MHz}, \mathrm{CDCl}_{3}\right) \delta=8.22$ $(\mathrm{d}, J=8 \mathrm{~Hz}, 1 \mathrm{H}), 8.06(\mathrm{~d}, J=8 \mathrm{~Hz}, 1 \mathrm{H}), 7.90(\mathrm{t}, J=8 \mathrm{~Hz}, 1 \mathrm{H}), 7.63(\mathrm{t}, J=8 \mathrm{~Hz}, 1 \mathrm{H}), 7.20-$ $7.16(\mathrm{~m}, 2 \mathrm{H}), 6.89(\mathrm{~d}, J=8 \mathrm{~Hz}, 1 \mathrm{H}), 5.68(\mathrm{~s}, 2 \mathrm{H}), 3.91(\mathrm{~s}, 3 \mathrm{H}), 3.89(\mathrm{~s}, 3 \mathrm{H}) \cdot{ }^{13} \mathrm{C} \mathrm{NMR}(50$ $\left.\mathrm{MHz}, \mathrm{CDCl}_{3}\right) \delta=167.6,160.1,150.4,149.4,149.0,134.4,129.0,128.7,128.6,128.1,126.8$, 123.7, 122.2, 115.6, 112.6, 111.0, 97.3, 69.7, 56.0, 55.9. LC-MS (ESI+) $t_{R} 4.95 \mathrm{~min}, \mathrm{~m} / \mathrm{z}$ $[\mathrm{M}+\mathrm{H}]^{+}$413.00/414.75/416.93. MW: $413.68 \mathrm{~g} / \mathrm{mol}$. Anal. Calcd for $\mathrm{C}_{18} \mathrm{H}_{15} \mathrm{Cl}_{3} \mathrm{~N}_{2} \mathrm{O}_{3}$ : C, 52.26; H, 3.65; N, 6.77. Found: C, 52.09; H, 3.64; N, 6.72.

\subsubsection{4-(1-Phenylethoxy)-2-(trichloromethyl)quinazoline (12)}

Yield 35\%. Beige powder. Mp141 ${ }^{\circ} \mathrm{C}$, (isopropanol). ${ }^{1} \mathrm{H}$ NMR $\left(200 \mathrm{MHz}, \mathrm{CDCl}_{3}\right) \delta=8.28$ (dd, $J=1 \mathrm{~Hz} ; 8 \mathrm{~Hz}, 1 \mathrm{H}), 8.05$ (d, $J=8 \mathrm{~Hz}, 1 \mathrm{H}), 7.89$ (t, $J=8 \mathrm{~Hz}, 1 \mathrm{H}), 7.70-7.56(\mathrm{~m}, 3 \mathrm{H})$, 
7.41-7.29 (m, 3H), $6.58(\mathrm{q}, J=7 \mathrm{~Hz}, 1 \mathrm{H}), 1.84(\mathrm{~d}, J=7 \mathrm{~Hz}, 3 \mathrm{H}) \cdot{ }^{13} \mathrm{C}$ NMR $(50 \mathrm{MHz}$, $\left.\mathrm{CDCl}_{3}\right) \delta=167.1,160.1,150.4,141.2,135.5,134.3,129.2,129.0,128.8,128.5,128.1,126.9$, 126.8, 123.7, 115.7, 97.2, 76.0, 22.1. LC-MS (ESI+) $t_{R} \quad 5.83 \quad \mathrm{~min}, \mathrm{~m} / \mathrm{z}$ $[\mathrm{M}+\mathrm{H}]^{+} 366.97 / 368.97 / 371.04$. MW: $367.66 \mathrm{~g} / \mathrm{mol}$. Anal. Calcd for $\mathrm{C}_{17} \mathrm{H}_{13} \mathrm{Cl}_{3} \mathrm{~N}_{2} \mathrm{O}: \mathrm{C}, 55.54$; H, 3.56; N, 7.62. Found: C, 55.59; H, 3.59; N, 7.71.

\subsubsection{4-(1-(4-Chlorophenyl)ethoxy)-2-(trichloromethyl)quinazoline (13)}

Yield 68\%. Brown powder. $\mathrm{Mp} 86{ }^{\circ} \mathrm{C}$, (isopropanol). ${ }^{1} \mathrm{H}$ NMR $\left(200 \mathrm{MHz}, \mathrm{CDCl}_{3}\right) \delta=8.22$ $(\mathrm{d}, J=8 \mathrm{~Hz}, 1 \mathrm{H}), 8.05(\mathrm{~d}, J=8 \mathrm{~Hz}, 1 \mathrm{H}), 7.90(\mathrm{td}, J=1 \mathrm{~Hz} ; 7 \mathrm{~Hz}, 1 \mathrm{H}), 7.66(\mathrm{td}, J=1 \mathrm{~Hz} ; 7$ $\mathrm{Hz}, 1 \mathrm{H}), 7.52(\mathrm{~d}, J=8 \mathrm{~Hz}, 2 \mathrm{H}), 7.32(\mathrm{~d}, J=8 \mathrm{~Hz}, 2 \mathrm{H}), 6.53(\mathrm{q}, J=7 \mathrm{~Hz}, 1 \mathrm{H}), 1.82$ (d, $J=7$ $\mathrm{Hz}, 3 \mathrm{H}) .{ }^{13} \mathrm{C} \mathrm{NMR}\left(50 \mathrm{MHz}, \mathrm{CDCl}_{3}\right) \delta=166.9,160.0,150.4,139.7,134.4,133.9128 .8$, 128.7, 128.3, 123.5, 115.6, 97.1, 75.2, 22.0. LC-MS (ESI+) $t_{R} 5.83 \mathrm{~min}, \mathrm{~m} / \mathrm{z}$ $[\mathrm{M}+\mathrm{H}]^{+} 400.96 / 402.81 / 404.83$. MW: $402.10 \mathrm{~g} / \mathrm{mol}$. Anal. Calcd for $\mathrm{C}_{17} \mathrm{H}_{12} \mathrm{Cl}_{4} \mathrm{~N}_{2} \mathrm{O}: \mathrm{C}, 50.78$; H, 3.01; N, 6.97. Found: C, 50.48; H, 3.03; N, 6.90 .

\subsubsection{4-(1-(2,4-Dichlorophenyl)ethoxy)-2-(trichloromethyl)quinazoline (14)}

Yield 69\%. Brown powder. Mp123 ${ }^{\circ} \mathrm{C}$, (isopropanol). ${ }^{1} \mathrm{H}$ NMR $\left(200 \mathrm{MHz}, \mathrm{CDCl}_{3}\right) \delta=8.31$ (d, $J=8 \mathrm{~Hz}, 1 \mathrm{H}), 8.07$ (d, $J=8 \mathrm{~Hz}, 1 \mathrm{H}), 7.96-7.87$ (m, 1H), 7.69 (t, $J=7 \mathrm{~Hz}, 1 \mathrm{H}), 7.49-7.39$ $(\mathrm{m}, 2 \mathrm{H}), 7.20(\mathrm{dd}, J=2 \mathrm{~Hz} ; 8 \mathrm{~Hz}, 1 \mathrm{H}), 6.78(\mathrm{q}, J=7 \mathrm{~Hz}, 1 \mathrm{H}), 1.81(\mathrm{~d}, J=7 \mathrm{~Hz}, 3 \mathrm{H}) \cdot{ }^{13} \mathrm{C}$ NMR $\left(50 \mathrm{MHz}, \mathrm{CDCl}_{3}\right) \delta=165.3,159.2,150.8,135.3,131.6,130.8$ 129.4, 129.3, 129.0, 128.6, 123.4, 115.4, 96.9, 72.7, 21.0.LC-MS (ESI+) $t_{R} 6.02 \quad \mathrm{~min}, \quad \mathrm{~m} / \mathrm{z}$ $[\mathrm{M}+\mathrm{H}]^{+} 434.74 / 436.86 / 438.81$. MW: $436.55 \mathrm{~g} / \mathrm{mol}$. Anal. Calcd for $\mathrm{C}_{17} \mathrm{H}_{11} \mathrm{Cl}_{5} \mathrm{~N}_{2} \mathrm{O}: \mathrm{C}, 46.77$; H, 2.54; N, 6.42. Found: C, 46.41; H, 2.52; N, 6.51.

\subsubsection{4-(1-Phenylprop-2-ynyloxy)-2-(trichloromethyl)quinazoline (15)}

Yield 84\%. Pale yellow powder. Mp $120{ }^{\circ} \mathrm{C}$, (isopropanol). ${ }^{1} \mathrm{H}$ NMR $\left(200 \mathrm{MHz}, \mathrm{CDCl}_{3}\right) \delta=$ 8.28-8.24 (m, 1H), 8.07 (d, $J=8 \mathrm{~Hz}, 1 \mathrm{H}), 7.95-7.87$ (m, 1H), 7.82-7.77 (m, 2H), 7.69-7.61 $(\mathrm{m}, 1 \mathrm{H}), 7.47-7.39(\mathrm{~m}, 3 \mathrm{H}), 7.26-7.24(\mathrm{~m}, 1 \mathrm{H}), 2.75(\mathrm{~d}, J=2 \mathrm{~Hz}, 1 \mathrm{H}) .{ }^{13} \mathrm{C} \mathrm{NMR}(50 \mathrm{MHz}$, $\left.\mathrm{CDCl}_{3}\right) \delta=166.3,159.7,150.6,136.3,134.7$ 129.4, 128.8, 128.7, 128.4, 123.8, 115.4, 97.0, 80.1, 76.4, 68.6.LC-MS (ESI+) $t_{R} 5.20 \mathrm{~min}, \mathrm{~m} / \mathrm{z}[\mathrm{M}+\mathrm{H}]^{+} 377.11 / 379.05 / 381.08$. MW: 377.65 g/mol. Anal. Calcd for $\mathrm{C}_{18} \mathrm{H}_{11} \mathrm{Cl}_{3} \mathrm{~N}_{2} \mathrm{O}: \mathrm{C}, 57.25 ; \mathrm{H}, 2.94 ; \mathrm{N}, 7.42$. Found: $\mathrm{C}, 57.61 ; \mathrm{H}, 2.95$; N, 7.35. 
Yield 41\%. Pale yellow powder. Mp $141{ }^{\circ} \mathrm{C}$, (isopropanol). ${ }^{1} \mathrm{H}$ NMR $\left(200 \mathrm{MHz}, \mathrm{CDCl}_{3}\right) \delta=$ 8.36-8.21 (m, 1H), $8.14(\mathrm{~d}, J=8 \mathrm{~Hz}, 1 \mathrm{H}), 7.99$ (td, $J=2 \mathrm{~Hz} ; 8 \mathrm{~Hz}, 1 \mathrm{H}), 7.80-7.68(\mathrm{~m}, 3 \mathrm{H})$,

7.54-7.49 (m, 3H), $7.14(\mathrm{~s}, 1 \mathrm{H}) .{ }^{13} \mathrm{C} \mathrm{NMR}\left(50 \mathrm{MHz}, \mathrm{CDCl}_{3}\right) \delta=166.3,159.7,150.6,136.3$, 134.7 129.4, 128.8, 128.7, 128.4, 123.8, 116.0, 114.7, 96.5, 65.9.LC-MS (ESI+) $t_{R} 4.73 \mathrm{~min}$, $\mathrm{m} / \mathrm{z}[\mathrm{M}+\mathrm{H}]^{+} 377.99 / 380.01 / 381.99$. MW: $378.64 \mathrm{~g} / \mathrm{mol}$. Anal. Calcd for $\mathrm{C}_{17} \mathrm{H}_{10} \mathrm{Cl}_{3} \mathrm{~N}_{3} \mathrm{O}: \mathrm{C}$, 53.93; H, 2.66; N, 11.10. Found: C, 53.89; H, 2.70; N, 11.12.

\subsubsection{4-((6-Nitrobenzo[d][1,3]dioxol-5-yl)methoxy)-2- (trichloromethyl)quinazoline (17)}

Yield 85\%. Pale yellow powder. Mp $181{ }^{\circ} \mathrm{C}$, (isopropanol). ${ }^{1} \mathrm{H}$ NMR $\left(200 \mathrm{MHz}, \mathrm{CDCl}_{3}\right) \delta=$ $8.25(\mathrm{~d}, J=8 \mathrm{~Hz}, 1 \mathrm{H}), 8.10$ (d, $J=8 \mathrm{~Hz}, 1 \mathrm{H}), 7.98-7.92(\mathrm{~m}, 1 \mathrm{H}), 7.74-7.65$ (m, 2H), 7.27-7.24 $(\mathrm{m}, 1 \mathrm{H}), 6.13(\mathrm{~s}, 2 \mathrm{H}), 6.08(\mathrm{~s}, 2 \mathrm{H}) .{ }^{13} \mathrm{C} \mathrm{NMR}\left(50 \mathrm{MHz}, \mathrm{CDCl}_{3}\right) \delta=167.1,159.9,152.2,150.5$, 147.8, 142.3, 134.8 129.0, 128.9, 123.5, 115.3, 109.1, 106.0, 103.2, 96.9, 66.2.LC-MS (ESI+) $t_{R} 5.00 \mathrm{~min}, \mathrm{~m} / \mathrm{z}[\mathrm{M}+\mathrm{H}]^{+} 441.87 / 443.97 / 445.95$. MW: $442.64 \mathrm{~g} / \mathrm{mol}$. Anal. Calcd for $\mathrm{C}_{17} \mathrm{H}_{10} \mathrm{Cl}_{3} \mathrm{~N}_{3} \mathrm{O}_{5}:$ C, 46.13; H, 2.28; N, 9.49. Found: C, 45.91; H, 2.27; N, 9.57.

\subsubsection{0.(E)-4-(3-(4-Nitrophenyl)allyloxy)-2-(trichloromethyl)quinazoline (18)}

Yield 45\%. Yellow powder. Mp $140{ }^{\circ} \mathrm{C}$, (isopropanol). ${ }^{1} \mathrm{H}$ NMR $\left(200 \mathrm{MHz}, \mathrm{CDCl}_{3}\right) \delta=8.29$ (m, 4H), 7.98-7.89(m, 1H), 7.73-7.65 (m, 1H), 7.58-7.24 (m, 2H), $6.98(\mathrm{~d}, J=16 \mathrm{~Hz}, 1 \mathrm{H})$, 6.80-6.66 (m, 1H), $5.41(\mathrm{dd}, J=1 \mathrm{~Hz} ; 6 \mathrm{~Hz}, 2 \mathrm{H}) .{ }^{13} \mathrm{C} \mathrm{NMR}\left(50 \mathrm{MHz}, \mathrm{CDCl}_{3}\right) \delta=167.3$, 160.0, 150.4, 147.4, 142.6, 134.6, 133.0, 131.9, 129.6, 129.1, 128.9, 128.8 127.6, 127.3, 124.1 123.8, 123.5, 115.4, 96.3, 67.7.LC-MS (ESI+) $t_{R} 5.34 \mathrm{~min}, \mathrm{~m} / \mathrm{z}[\mathrm{M}+\mathrm{H}]^{+} 423.94 / 425.92 / 427.96$. MW: $424.67 \mathrm{~g} / \mathrm{mol}$. Anal. Calcd for $\mathrm{C}_{18} \mathrm{H}_{12} \mathrm{Cl}_{3} \mathrm{~N}_{3} \mathrm{O}_{3}$ : C, 50.91; H, 2.85; N, 9.89. Found: C, 51.08; H, 2.86; N, 9.75.

\subsubsection{4-(3-(Benzyloxy)benzyloxy)-2-(trichloromethyl)quinazoline (19)}

Yield 68\%. Yellow powder. Mp $96{ }^{\circ} \mathrm{C}$, (isopropanol). ${ }^{1} \mathrm{H}$ NMR $\left(200 \mathrm{MHz}, \mathrm{CDCl}_{3}\right) \delta=$ 8.23(dd, $J=1 \mathrm{~Hz} ; 8 \mathrm{~Hz}, 1 \mathrm{H}), 8.10(\mathrm{~d}, J=8 \mathrm{~Hz}, 1 \mathrm{H}), 7.91$ (td, $J=1 \mathrm{~Hz} ; 8 \mathrm{~Hz}, 1 \mathrm{H}), 7.69-7.61$ $(\mathrm{m}, 1 \mathrm{H}), 7.47-7.18(\mathrm{~m}, 8 \mathrm{H}), 5.72(\mathrm{~s}, 1 \mathrm{H}), 5.72(\mathrm{~s}, 2 \mathrm{H}), 5.10(\mathrm{~s}, 2 \mathrm{H}) .{ }^{13} \mathrm{C}$ NMR $(50 \mathrm{MHz}$, $\left.\mathrm{CDCl}_{3}\right) \delta=167.6,160.1,159.0,150.4,137.2,136.9,134.5,130.0,129.7,128.9,128.8,128.6$, 128.0, 127.5, 123.7, 121.4, 115.5, 115.3, 115.0, 97.2, 70.1, 69.3.LC-MS (ESI+) $t_{R} 5.87 \mathrm{~min}$, $\mathrm{m} / \mathrm{z}[\mathrm{M}+\mathrm{H}]^{+} 458.74 / 460.72 / 462.84$. MW: $459.75 \mathrm{~g} / \mathrm{mol}$. Anal. Calcd for $\mathrm{C}_{23} \mathrm{H}_{17} \mathrm{Cl}_{3} \mathrm{~N}_{2} \mathrm{O}_{2}: \mathrm{C}$, 60.09; H, 3.73; N, 6.09. Found: C, 59.87; H, 3.70; N, 5.98.

\subsubsection{4-(4-Bromophenoxy)-2-(trichloromethyl)quinazoline (20)}


Yield 99\%. White powder. Mp $179{ }^{\circ} \mathrm{C}$, (isopropanol). ${ }^{1} \mathrm{H}$ NMR $\left(200 \mathrm{MHz}, \mathrm{CDCl}_{3}\right) \delta=8.42$ 8.38 (m, 1H), 8.16(d, $J=8 \mathrm{~Hz}, 1 \mathrm{H}), 8.02$ (td, $J=1 \mathrm{~Hz} ; 8 \mathrm{~Hz}, 1 \mathrm{H}), 7.81-7.73$ (m, 1H), 7.59 (d, $J=8 \mathrm{~Hz}, 2 \mathrm{H}), 7.30(\mathrm{~d}, J=8 \mathrm{~Hz}, 2 \mathrm{H}) .{ }^{13} \mathrm{C} \mathrm{NMR}\left(50 \mathrm{MHz}, \mathrm{CDCl}_{3}\right) \delta=164.4,157.5,148.7$, 148.6, 132.6, 130.0,126.8, 126.6, 121.1, 120.9, 112.8, 96.7.LC-MS (ESI+) $t_{R} 5.38 \mathrm{~min}, \mathrm{~m} / \mathrm{z}$ $[\mathrm{M}+\mathrm{H}]^{+} 418.26 / 420.82 / 422.93$. MW: $418.5 \mathrm{~g} / \mathrm{mol}$. Anal. Calcd for $\mathrm{C}_{15} \mathrm{H}_{8} \mathrm{BrCl}_{3} \mathrm{~N}_{2} \mathrm{O}: \mathrm{C}, 43.05$; H, 1.93; N, 6.69. Found: C, 43.25; H, 1.95; N, 6.58.

\subsubsection{4-(3-Bromophenoxy)-2-(trichloromethyl)quinazoline (21)}

Yield 99\%. White powder. Mp $114{ }^{\circ} \mathrm{C}$, (isopropanol). ${ }^{1} \mathrm{H}$ NMR $\left(200 \mathrm{MHz}, \mathrm{CDCl}_{3}\right) \delta=8.41-$ $8.37(\mathrm{~m}, 1 \mathrm{H}), 8.18-8.14(\mathrm{~m}, 1 \mathrm{H}), 8.01(\mathrm{td}, J=1 \mathrm{~Hz} ; 8 \mathrm{~Hz}, 1 \mathrm{H}), 7.81-7.73$ (m, 1H), 7.63-7.62 $(\mathrm{m}, 1 \mathrm{H}), 7.48-7.30(\mathrm{~m}, 3 \mathrm{H}) .{ }^{13} \mathrm{C} \mathrm{NMR}\left(50 \mathrm{MHz}, \mathrm{CDCl}_{3}\right) \delta=166.8,160.0,152.5,151.2,135.1$, 130.5, 129.3, 129.1, 125.1, 123.6, 122.3, 120.4, 115.2, 96.6.LC-MS (ESI+) $t_{R} 5.43 \mathrm{~min}, \mathrm{~m} / \mathrm{z}$ $[\mathrm{M}+\mathrm{H}]^{+} 418.18 / 420.78 / 422.82$. MW: $418.5 \mathrm{~g} / \mathrm{mol}$. Anal. Calcd for $\mathrm{C}_{15} \mathrm{H}_{8} \mathrm{BrCl}_{3} \mathrm{~N}_{2} \mathrm{O}: \mathrm{C}, 43.05$; H, 1.93; N, 6.69. Found: C, 42.91; H, 1.92; N, 6.76.

\subsubsection{4-(2-Bromophenoxy)-2-(trichloromethyl)quinazoline (22)}

Yield 99\%. White powder. Mp $138{ }^{\circ} \mathrm{C}$, (isopropanol). ${ }^{1} \mathrm{H}$ NMR $\left(200 \mathrm{MHz}, \mathrm{CDCl}_{3}\right) \delta=8.51$ $8.47(\mathrm{~m}, 1 \mathrm{H}), 8.17$ (d, $J=8 \mathrm{~Hz}, 1 \mathrm{H}), 8.02$ (td, $J=1 \mathrm{~Hz} ; 8 \mathrm{~Hz}, 1 \mathrm{H}), 7.84-7.67$ (m, 2H), 7.49$7.39(\mathrm{~m}, 2 \mathrm{H}), 7.28-7.17(\mathrm{~m}, 1 \mathrm{H}) .{ }^{13} \mathrm{C} \mathrm{NMR}\left(50 \mathrm{MHz}, \mathrm{CDCl}_{3}\right) \delta=166.7$, 159.9, 151.1, 149.4, 135.0, 133.4, 129.2, 128.9, 128.4, 127.4, 123.8, 123.7, 116.2, 114.9, 96.5. LC-MS (ESI+) $t_{R}$ $5.05 \mathrm{~min}, \mathrm{~m} / \mathrm{z}[\mathrm{M}+\mathrm{H}]^{+}$418.07/420.73/422.87. MW: $418.5 \mathrm{~g} / \mathrm{mol}$. Anal. Calcd for $\mathrm{C}_{15} \mathrm{H}_{8} \mathrm{BrCl}_{3} \mathrm{~N}_{2} \mathrm{O}: \mathrm{C}, 43.05 ; \mathrm{H}, 1.93 ; \mathrm{N}, 6.69$. Found: $\mathrm{C}, 42.89 ; \mathrm{H}, 1.91 ; \mathrm{N}, 6.81$.

\subsubsection{4-(2-(Trichloromethyl)quinazolin-4-yloxy)benzonitrile (23)}

Yield 38\%. White powder. Mp194 ${ }^{\circ} \mathrm{C}$, (isopropanol). ${ }^{1} \mathrm{H}$ NMR $\left(200 \mathrm{MHz}, \mathrm{CDCl}_{3}\right) \delta=8.41$ $(\mathrm{d}, J=8 \mathrm{~Hz}, 1 \mathrm{H}), 8.19(\mathrm{~d}, J=8 \mathrm{~Hz}, 1 \mathrm{H}), 8.07-8.00(\mathrm{~m}, 1 \mathrm{H}), 7.84-7.78(\mathrm{~m}, 3 \mathrm{H}), 7.57(\mathrm{~d}, J=8$ $\mathrm{Hz}, 2 \mathrm{H}) .{ }^{13} \mathrm{C}$ NMR $\left(50 \mathrm{MHz}, \mathrm{CDCl}_{3}\right) \delta=166.4,159.7,155.4,151.3,135.4,133.8,129.6$, $129.2,123.5,122.7,118.3,115.0,109.9$, 96.5. LC-MS (ESI+) $t_{R} 4.46 \mathrm{~min}, \mathrm{~m} / \mathrm{z}[\mathrm{M}+\mathrm{H}]^{+}$ 364.10/365.99/367.99. MW: $364.61 \mathrm{~g} / \mathrm{mol}$. Anal. Calcd for $\mathrm{C}_{16} \mathrm{H}_{8} \mathrm{Cl}_{3} \mathrm{~N}_{3} \mathrm{O}$ : C, 52.71; $\mathrm{H}, 2.21$; N, 11.52. Found :C, 52.71; H, 2.21; N, 11.52.

\subsubsection{1-(4-((2-(Trichloromethyl)quinazolin-4-yl)oxy)phenyl)ethanone (24)}

Yield 95\%. White powder. Mp $149{ }^{\circ} \mathrm{C}$, (isopropanol). ${ }^{1} \mathrm{H}$ NMR $\left(200 \mathrm{MHz}, \mathrm{CDCl}_{3}\right) \delta=8.41$ (d, $J=8 \mathrm{~Hz}, 1 \mathrm{H}), 8.18-7.97(\mathrm{~m}, 4 \mathrm{H}), 7.78(\mathrm{t}, J=8 \mathrm{~Hz}, 1 \mathrm{H}), 7.52(\mathrm{~d}, J=8 \mathrm{~Hz}, 2 \mathrm{H}), 2.65$ (s, $3 \mathrm{H}) .{ }^{13} \mathrm{C} \mathrm{NMR}\left(50 \mathrm{MHz}, \mathrm{CDCl}_{3}\right) \delta=196.9,166.7,159.9,155.8,151.2,135.2,134.7,130.0$, 
129.4, 129.1, 123.6, 121.6, 115.2, 96.6, 26.7. LC-MS (ESI+) $t_{R} 4.48 \mathrm{~min}, \mathrm{~m} / \mathrm{z}$ $[\mathrm{M}+\mathrm{H}]^{+} 381.07 / 383.07 / 385.02$. MW: $381.64 \mathrm{~g} / \mathrm{mol}$. Anal. Calcd for $\mathrm{C}_{17} \mathrm{H}_{11} \mathrm{Cl}_{3} \mathrm{~N}_{2} \mathrm{O}_{2}: \mathrm{C}, 53.50$; H, 2.91; N, 7.34. Found :C, 53.71; H, 2.89; N, 7.41.

\subsubsection{1-(3-(2-(Trichloromethyl)quinazolin-4-yloxy)phenyl)ethanone (25)}

Yield 99\%. White powder. Mp $106{ }^{\circ} \mathrm{C}$, (isopropanol). ${ }^{1} \mathrm{H}$ NMR $\left(200 \mathrm{MHz}, \mathrm{CDCl}_{3}\right) \delta=8.43$ $(\mathrm{dd}, J=1 \mathrm{~Hz} ; 8 \mathrm{~Hz}, 1 \mathrm{H}), 8.17$ (d, $J=8 \mathrm{~Hz}, 1 \mathrm{H}), 8.05-7.89(\mathrm{~m}, 3 \mathrm{H}), 7.83-7.75$ (m, 1H), 7.67$7.54(\mathrm{~m}, 2 \mathrm{H}), 2.64(\mathrm{~s}, 3 \mathrm{H}) .{ }^{13} \mathrm{C} \mathrm{NMR}\left(50 \mathrm{MHz}, \mathrm{CDCl}_{3}\right) \delta=197.1,167.0,159.9,151.1,138.5$, $135.1,129.7,129.3,129.0,126.3,125.8,123.6,121.5,115.2,96.7,26.8$. LC-MS (ESI+) $t_{R}$ $4.47 \mathrm{~min}, \mathrm{~m} / \mathrm{z}[\mathrm{M}+\mathrm{H}]^{+}$381.07/383.05/384.98. MW: $381.64 \mathrm{~g} / \mathrm{mol}$. Anal. Calcd for $\mathrm{C}_{17} \mathrm{H}_{11} \mathrm{Cl}_{3} \mathrm{~N}_{2} \mathrm{O}_{2}$ : C, 53.50; H, 2.91; N, 7.34. Found :C, 53.21; H, 2.90; N, 7.42.

\subsubsection{1-(2-(2-(Trichloromethyl)quinazolin-4-yloxy)phenyl)ethanone (26)}

Yield 47\%. White powder. Mp $147{ }^{\circ} \mathrm{C}$, (isopropanol). ${ }^{1} \mathrm{H}$ NMR (200 MHz, $\left.\mathrm{CDCl}_{3}\right) \delta=8.46$ (d, $J=8 \mathrm{~Hz}, 1 \mathrm{H}), 8.12$ (d, $J=8 \mathrm{~Hz}, 1 \mathrm{H}), 8.05-7.89$ (m, 2H), 7.83-7.75 (m, 1H), 7.68-7.59(m, 1H), 7.46-7.33 (m, 2H), 2.50 (s, 3H). ${ }^{13} \mathrm{C} \mathrm{NMR}\left(50 \mathrm{MHz}, \mathrm{CDCl}_{3}\right) \delta=197.4,167.5,159.9$, $151.2,150.6,135.1,133.6,131.3,130.4,129.4,129.0,126.3,123.7,115.2$, 96.8, 29.7. LCMS (ESI+) $t_{R} 4.16 \mathrm{~min}, \mathrm{~m} / \mathrm{z}[\mathrm{M}+\mathrm{H}]^{+} 381.03 / 383.03 / 384.99$. MW: $381.64 \mathrm{~g} / \mathrm{mol}$. Anal. Calcd for $\mathrm{C}_{17} \mathrm{H}_{11} \mathrm{Cl}_{3} \mathrm{~N}_{2} \mathrm{O}_{2}:$ C, 53.50; H, 2.91; N, 7.34. Found :C, 53.27; H, 2.93; N, 7.29.

\subsubsection{4-(3-Methoxyphenoxy)-2-trichloromethylquinazoline (27)}

Yield 98\%. White powder. Mp $101{ }^{\circ} \mathrm{C}$ (Lit. $101{ }^{\circ} \mathrm{C}$ [19]), (isopropanol). ${ }^{1} \mathrm{H}$ NMR (200 MHz, $\left.\mathrm{CDCl}_{3}\right) \delta=8.39-8.43(\mathrm{~m}, 1 \mathrm{H}), 8.13-8.17(\mathrm{~m}, 1 \mathrm{H}), 7.95-8.03(\mathrm{~m}, 1 \mathrm{H}), 7.72-7.80(\mathrm{~m}, 1 \mathrm{H})$, 7.32-7.40 (m, 1H), 6.96-7.05 (m, 2H), 6.83-6.89 (m, 1H), $3.83(\mathrm{~s}, 3 \mathrm{H}) .{ }^{13} \mathrm{C}$ NMR $(50 \mathrm{MHz}$, $\left.\mathrm{CDCl}_{3}\right) \delta=167.4,160.4,160.1,153.1,151.0,134.8,129.7,129.1,128.9,123.7,115.4,113.5$, 112.0, 107.2, 96.8, 55.5 LC-MS (ESI+) $t_{R} 5.10 \mathrm{~min}, \mathrm{~m} / \mathrm{z}[\mathrm{M}+\mathrm{H}]^{+} 369.01 / 371.15 / 373.09$. MW: $369.63 \mathrm{~g} / \mathrm{mol}$. Anal. Calcd for $\mathrm{C}_{16} \mathrm{H}_{11} \mathrm{Cl}_{3} \mathrm{~N}_{2} \mathrm{O}_{2}$ :C, 51.99; H, 3.00; N, 7.58.Found :C, 52.16; H, $3.07 ; \mathrm{N}, 7.67$.

\subsubsection{2-(2-(Trichloromethyl)quinazolin-4-yloxy)benzaldehyde (28)}

Yield $78 \%$. White powder. Mp $111{ }^{\circ} \mathrm{C}$, (isopropanol). ${ }^{1} \mathrm{H}$ NMR $\left(200 \mathrm{MHz}, \mathrm{CDCl}_{3}\right) \delta=10.19$ (s, 1H), 8.47 (dd, $J=1 \mathrm{~Hz} ; 8 \mathrm{~Hz}, 1 \mathrm{H}), 8.17$ (d, $J=8 \mathrm{~Hz}, 1 \mathrm{H}), 8.06-7.98$ (m, 2H), 7.87-7.69 
(m, 2H), 7.52-7.45 (m, 2H). $\left.{ }^{13} \mathrm{C} \mathrm{NMR} \mathrm{(50} \mathrm{MHz,} \mathrm{CDCl}_{3}\right) \delta=188.4,167.5,163.1,153.3$, $151.2,146.7,135.5,130.1,129.5,129.1,126.7,123.6,123.3,120.9,114.9,96.5$. LC-MS $(\mathrm{ESI}+) t_{R} 4.20 \mathrm{~min}, \mathrm{~m} / \mathrm{z}[\mathrm{M}+\mathrm{H}]^{+} 367.03 / 369.02 / 371.03$. MW: $367.61 \mathrm{~g} / \mathrm{mol}$. Anal. Calcd for $\mathrm{C}_{16} \mathrm{H}_{9} \mathrm{Cl}_{3} \mathrm{~N}_{2} \mathrm{O}_{2}:$ C, 52.28; H, 2.47; N, 7.62. Found : C, 52.59; H, 2.45; N, 7.68.

\subsubsection{4-(Perfluorophenoxy)-2-(trichloromethyl)quinazoline (29)}

Yield 94\%. White powder. Mp $139{ }^{\circ} \mathrm{C}$, (isopropanol). ${ }^{1} \mathrm{H}$ NMR $\left(200 \mathrm{MHz}, \mathrm{CDCl}_{3}\right) \delta=8.43$ $(\mathrm{dd}, J=1 \mathrm{~Hz} ; 8 \mathrm{~Hz}, 1 \mathrm{H}), 8.22$ (d, $J=8 \mathrm{~Hz}, 1 \mathrm{H}), 8.07$ (td, $J=1 \mathrm{~Hz} ; 8 \mathrm{~Hz}, 1 \mathrm{H}), 7.88-7.80$ (m, 1H). ${ }^{13} \mathrm{C} \mathrm{NMR}\left(50 \mathrm{MHz}, \mathrm{CDCl}_{3}\right) \delta=165.4,159.5,151.4,135.7,129.9,129.2,123.4,114.0$, 96.0. LC-MS (ESI+) $t_{R} 5.49 \mathrm{~min}, \mathrm{~m} / \mathrm{z}[\mathrm{M}+\mathrm{H}]^{+}$428.71/430.78/432.67.MW: $429.56 \mathrm{~g} / \mathrm{mol}$. Anal. Calcd for $\mathrm{C}_{15} \mathrm{H}_{4} \mathrm{Cl}_{3} \mathrm{~F}_{5} \mathrm{~N}_{2} \mathrm{O}: \mathrm{C}, 41.94 ; \mathrm{H}, 0.94 ; \mathrm{N}, 6.52$. Found :C, 42.12; H, 0.97; N, 6.42 .

3.1.32. 3-Methoxy-5-nitro-4-(2-(trichloromethyl)quinazolin-4-yloxy)benzaldehyde (30)

Yield 79\%. Yellow powder. Mp $171{ }^{\circ} \mathrm{C}$, (isopropanol). ${ }^{1} \mathrm{H} \mathrm{NMR}\left(200 \mathrm{MHz}, \mathrm{CDCl}_{3}\right) \delta=$ $10.28(\mathrm{~s}, 1 \mathrm{H}), 8.49-8.46(\mathrm{~m}, 2 \mathrm{H}), 8.24-8.05(\mathrm{~m}, 3 \mathrm{H}), 7.86(\mathrm{t}, J=6 \mathrm{~Hz}, 1 \mathrm{H}), 3.86(\mathrm{~s}, 3 \mathrm{H}) .{ }^{13} \mathrm{C}$ NMR $\left(50 \mathrm{MHz}, \mathrm{CDCl}_{3}\right) \delta=186.2,166.6,159.6,152.8,151.4,148.0,146.5,135.6,135.1$, $129.8,129.6,129.3,123.5,115.6,114.3,111.5,96.2,56.9$. LC-MS (ESI+) $t_{R} 4.01 \mathrm{~min}, \mathrm{~m} / \mathrm{z}$ $[\mathrm{M}+\mathrm{H}]^{+}$441.87/443.71/445.72.MW: $442.64 \mathrm{~g} / \mathrm{mol}$. Anal. Calcd for $\mathrm{C}_{17} \mathrm{H}_{10} \mathrm{Cl}_{3} \mathrm{~N}_{3} \mathrm{O}_{5}: \mathrm{C}, 46.13$; H, 2.28; N, 9.49. Found : C, 45.95; H, 2.30; N, 9.55.

\subsubsection{4-(4-Chloro-3-methylphenoxy)-2-(trichloromethyl)quinazoline (31)}

Yield 92\%. White powder. Mp $98{ }^{\circ} \mathrm{C}$, (isopropanol). ${ }^{1} \mathrm{H}$ NMR $\left(200 \mathrm{MHz}, \mathrm{CDCl}_{3}\right) \delta=8.37$ $(\mathrm{dd}, J=1 \mathrm{~Hz} ; 8 \mathrm{~Hz}, 1 \mathrm{H}), 8.16-8.12(\mathrm{~m}, 1 \mathrm{H}), 8.02-7.94(\mathrm{~m}, 1 \mathrm{H}), 7.79-7.70$ (m, 1H), 7.41 (d, $J$ $=8 \mathrm{~Hz}, 1 \mathrm{H}), 7.31-7.30(\mathrm{~m}, 1 \mathrm{H}), 7.20(\mathrm{dd}, J=1 \mathrm{~Hz} ; 8 \mathrm{~Hz}, 1 \mathrm{H}), 2.41(\mathrm{~s}, 3 \mathrm{H}) .{ }^{13} \mathrm{C} \mathrm{NMR}(50$ $\left.\mathrm{MHz}, \mathrm{CDCl}_{3}\right) \delta=166.9,159.9,151.0,150.4,137.3,134.9,131.1,129.6,129.1,128.9,123.7$, 123.5, 120.1, 115.2, 96.7, 20.3. MW: $388.08 \mathrm{~g} / \mathrm{mol}$. HRMS (ESI): $m / z$ [M + Na $]^{+}$calcd for $\left[\mathrm{C}_{16} \mathrm{H}_{10} \mathrm{Cl}_{4} \mathrm{~N}_{2} \mathrm{O}\right]^{+}$: 408.94395 ; found: 408.94429.

\subsubsection{4-[(2,4-Dichlorophenyl)oxy]-2-(trichloromethyl)quinazoline (32)}

Yield 89\%. White powder. Mp $110{ }^{\circ} \mathrm{C}$, (isopropanol). ${ }^{1} \mathrm{H} \mathrm{NMR}\left(200 \mathrm{MHz}, \mathrm{CDCl}_{3}\right) \delta=8.43$ $8.48(\mathrm{~m}, 1 \mathrm{H}), 8.18$ (d, $J=8 \mathrm{~Hz}, 1 \mathrm{H}), 8.06-7.98$ (m, 1H), 7.84-7.75 (m, 1H), 7.54-7.33 (m, $3 \mathrm{H}) .{ }^{13} \mathrm{C} \mathrm{NMR}\left(50 \mathrm{MHz}, \mathrm{CDCl}_{3}\right) \delta=166.5,159.8,151.2,147.0,135.2,131.9,130.2,129.4$, 129.0, 128.0, 127.9, 124.6, 123.6, 114.8, 96.4. LC-MS (ESI+) $t_{R} 4.60 \mathrm{~min}, \mathrm{~m} / \mathrm{z}[\mathrm{M}+\mathrm{H}]^{+}$ 
408.05/410.09/411.98.MW: $408.49 \mathrm{~g} / \mathrm{mol}$. Anal. Calcd for $\mathrm{C}_{15} \mathrm{H}_{7} \mathrm{Cl}_{5} \mathrm{~N}_{2} \mathrm{O}: \mathrm{C}, 44.10 ; \mathrm{H}, 1.73$; N, 6.86. Found : C, 44.50; H, 1.71; N, 6.97.

\subsubsection{2-Methyl-3-(2-(trichloromethyl)quinazolin-4-yloxy)-4H-pyran-4-one (33)}

Yield 67\%. Beige powder. Mp202 ${ }^{\circ} \mathrm{C}$, (isopropanol). ${ }^{1} \mathrm{H}$ NMR $\left(200 \mathrm{MHz}, \mathrm{CDCl}_{3}\right) \delta=8.41$ $(\mathrm{dd}, J=1 \mathrm{~Hz} ; 8 \mathrm{~Hz}, 1 \mathrm{H}), 8.15$ (d, $J=8 \mathrm{~Hz}, 1 \mathrm{H}), 7.99$ (td, $J=1 \mathrm{~Hz} ; 8 \mathrm{~Hz}, 1 \mathrm{H}), 7.82-7.72$ (m, $2 \mathrm{H}), 6.49(\mathrm{~d}, J=4 \mathrm{~Hz}, 1 \mathrm{H}), 2.36(\mathrm{~s}, 3 \mathrm{H}) .{ }^{13} \mathrm{C} \mathrm{NMR}\left(50 \mathrm{MHz}, \mathrm{CDCl}_{3}\right) \delta=171.9,165.9,159.8$, $154.4,151.3,135.4,135.1,129.2,128.9,126.8,123.8,117.0,113.0,96.5,15.2$. LC-MS (ESI+) $t_{R} 3.29 \mathrm{~min}, \mathrm{~m} / \mathrm{z}[\mathrm{M}+\mathrm{H}]^{+} 370.97 / 372.99 / 375.00 . \mathrm{MW}: 371.60 \mathrm{~g} / \mathrm{mol}$. Anal. Calcd for $\mathrm{C}_{15} \mathrm{H}_{9} \mathrm{Cl}_{3} \mathrm{~N}_{2} \mathrm{O}_{3}: \mathrm{C}, 48.48 ; \mathrm{H}, 2.44 ; \mathrm{N}, 7.54$. Found :C, 48.38; H, 2.41; N, 7.62.

\subsubsection{4-(5,7-Dibromoquinolin-8-yloxy)-2-(trichloromethyl)quinazoline (34)}

Yield 98\%. Brown powder. Mp186 ${ }^{\circ} \mathrm{C}$, (isopropanol). ${ }^{1} \mathrm{H}$ NMR $\left(200 \mathrm{MHz}, \mathrm{CDCl}_{3}\right) \delta=8.77$ $8.55(\mathrm{~m}, 3 \mathrm{H}), 8.21-8.12(\mathrm{~m}, 2 \mathrm{H}), 8.04(\mathrm{t}, J=8 \mathrm{~Hz}, 1 \mathrm{H}), 7.83(\mathrm{t}, J=6 \mathrm{~Hz}, 1 \mathrm{H}), 7.56-7.53(\mathrm{~m}$, 1H). ${ }^{13} \mathrm{C} \mathrm{NMR}\left(50 \mathrm{MHz}, \mathrm{CDCl}_{3}\right) \delta=167.13,159.6,151.4,151.3,146.5,142.2,136.2,135.1$, 133.3, 129.3, 128.9, 127.8, 124.1, 122.8, 119.5, 116.9, 114.8, 96.4. LC-MS (ESI+) $t_{R} 5.37 \mathrm{~min}$, $\mathrm{m} / \mathrm{z}[\mathrm{M}+\mathrm{H}]^{+} 546.08 / 547.52 / 549.54 . \mathrm{MW}: 548.44 \mathrm{~g} / \mathrm{mol}$. Anal. Calcd for $\mathrm{C}_{18} \mathrm{H}_{8} \mathrm{Br}_{2} \mathrm{~N}_{3} \mathrm{O}: \mathrm{C}$, 39.42; H, 1.47; N, 7.66. Found: C, 39.49; H, 1.50; N, 7.51.

\subsubsection{4-(Quinolin-2-yloxy)-2-(trichloromethyl)quinazoline (35)}

Yield 67\%. Yellow powder. Mp $163{ }^{\circ} \mathrm{C}$, (isopropanol). ${ }^{1} \mathrm{H}$ NMR $\left(200 \mathrm{MHz}, \mathrm{CDCl}_{3}\right) \delta=$ 8.52(d, $J=8 \mathrm{~Hz}, 1 \mathrm{H}), 8.34(\mathrm{~d}, J=8 \mathrm{~Hz}, 1 \mathrm{H}), 8.18(\mathrm{~d}, J=8 \mathrm{~Hz}, 1 \mathrm{H}), 8.07-7.72(\mathrm{~m}, 5 \mathrm{H}), 7.64-$ $7.49(\mathrm{~m}, 2 \mathrm{H}) .{ }^{13} \mathrm{C} \mathrm{NMR}\left(50 \mathrm{MHz}, \mathrm{CDCl}_{3}\right) \delta=166.9,159.8,157.4,151.3,146.3,140.1,135.3$, 130.5, 129.4, 129.0, 128.4, 127.3, 126.8, 124.0, 115.7, 115.2, 96.5. LC-MS (ESI+) $t_{R} 4.60 \mathrm{~min}$, $\mathrm{m} / \mathrm{z}[\mathrm{M}+\mathrm{H}]^{+} 389.95 / 391.95 / 394.06 . \mathrm{MW}: 390.65 \mathrm{~g} / \mathrm{mol}$. Anal. Calcd for $\mathrm{C}_{18} \mathrm{H}_{10} \mathrm{Cl}_{3} \mathrm{~N}_{3} \mathrm{O}: \mathrm{C}$, 55.34; H, 2.58; N, 10.76. Found :C, 55.13; H, 2.56; N, 10.91.

\subsubsection{4-(2-(Trichloromethyl)quinazolin-4-yloxy)-2H-chromen-2-one (36)}

Yield 55\%. Yellow powder. Mp237 ${ }^{\circ} \mathrm{C}$, (isopropanol). ${ }^{1} \mathrm{H}$ NMR $\left(200 \mathrm{MHz}, \mathrm{CDCl}_{3}\right) \delta=8.45$ $(\mathrm{d}, J=8 \mathrm{~Hz}, 1 \mathrm{H}), 8.26(\mathrm{~d}, J=8 \mathrm{~Hz}, 1 \mathrm{H}), 8.10(\mathrm{t}, J=8 \mathrm{~Hz}, 1 \mathrm{H}), 7.92-7.80(\mathrm{~m}, 2 \mathrm{H}), 7.68-7.60$ $(\mathrm{m}, 1 \mathrm{H}), 7.47-7.30(\mathrm{~m}, 2 \mathrm{H}), 6.96(\mathrm{~s}, 1 \mathrm{H}) .{ }^{13} \mathrm{C} \mathrm{NMR}\left(50 \mathrm{MHz}, \mathrm{CDCl}_{3}\right) \delta=165.3,161.6,159.7$, $153.7,151.7135 .9,133.0,130.1,129.6,124.4,123.1,122.9,117.3,115.5,115.1,104.3,96.1$. 
LC-MS (ESI+) $t_{R} 4.60 \mathrm{~min}, \mathrm{~m} / \mathrm{z}[\mathrm{M}+\mathrm{H}]^{+} 406.93 / 408.93 / 411.18 . \mathrm{MW}: 407.63 \mathrm{~g} / \mathrm{mol}$. Anal. Calcd for $\mathrm{C}_{18} \mathrm{H}_{9} \mathrm{Cl}_{3} \mathrm{~N}_{2} \mathrm{O}_{3}: \mathrm{C}, 53.04 ; \mathrm{H}, 2.23 ; \mathrm{N}, 6.87$. Found :C, 52.86; H, 2.24; N, 6.92.

\subsubsection{Preparation of 1-(2-(2-methylquinazolin-4-yloxy)phenyl)ethanone (37)}

Molecule 37 was prepared in two steps from commercial 2-methylquinazolin-4(3H)-one. This last was first chlorinated at position 4 , by using $\mathrm{POCl}_{3}$, according to a previously microwaveassisted reported procedure [25], leading to 4-chloro-2-methylquinazoline in 73\% yield. Then, the general DMAP-catalyzed operating procedure described in $\S$ 3.1.3. was applied to this intermediate product, leading to molecule 37 in $45 \%$ yield, as a beige powder. $\mathrm{Mp} 118{ }^{\circ} \mathrm{C}$, (isopropanol). ${ }^{1} \mathrm{H} \mathrm{NMR}\left(200 \mathrm{MHz}, \mathrm{CDCl}_{3}\right) \delta=8.33(\mathrm{~d}, J=8 \mathrm{~Hz}, 1 \mathrm{H}), 7.95-7.83(\mathrm{~m}, 3 \mathrm{H})$, 7.65-7.56 (m, 2H), 7.42-7.25 (m, 2H), 2.59 (s, 3H), 2.47 (s, 3H). $\left.{ }^{13} \mathrm{C} \mathrm{NMR} \mathrm{(50} \mathrm{MHz,} \mathrm{CDCl}_{3}\right)$ $\delta=197.9,166.3,163.7,152.0,150.9,134.3,133.3,132.0,130.1,127.1,127.0,126.0,123.8$, 123.4, 114.3, 30.1, 26.2. LC-MS (ESI+) $t_{R} 4.60 \mathrm{~min}, \mathrm{~m} / \mathrm{z}[\mathrm{M}+\mathrm{H}]^{+} 279.19 . \mathrm{MW}: 278.31 \mathrm{~g} / \mathrm{mol}$. Anal. Calcd for $\mathrm{C}_{17} \mathrm{H}_{14} \mathrm{~N}_{2} \mathrm{O}_{2}$ : C, 73.37; H, 5.07; N, 10.07. Found : C, 73.82; H, 5.11; N, 9.93.

\subsection{Biology}

\subsubsection{In vitroAntiplasmodial evaluation}

In this study, a K1 culture-adapted $P$. falciparum strain resistant to chloroquine, pyrimethamine and proguanil was used in an in vitro culture. Maintenance in continuous culture was done as described previously by Trager and Jensen [30]. Cultures were maintained in fresh A+ human erythrocytes at $2.5 \%$ hematocrit in complete medium (RPMI 1640 with $25 \mathrm{mM}$ HEPES, $25 \mathrm{mM} \mathrm{NaHCO} 3,10 \%$ of $\mathrm{A}+$ human serum) at $37{ }^{\circ} \mathrm{C}$ under reduced $\mathrm{O}_{2}$ atmosphere (gas mixture $14 \% \mathrm{O}_{2}, 6 \% \mathrm{CO}_{2}$, and $80 \% \mathrm{~N}_{2}$ ). Parasitaemia was maintained daily between $1 \%$ and $6 \%$. The P. falciparum drug susceptibility test was carried out by comparing quantities of DNA in treated and control cultures of parasite in human erythrocytes according to a SYBR Green I fluorescence-based method [31] using a 96-well fluorescence plate reader. Compounds, previously dissolved in DMSO (final concentration less than $0.5 \% \mathrm{v} / \mathrm{v}$ ) were incubated in a total assay volume of $200 \mu \mathrm{L}$ (RPMI, $4 \%$ hematocrit and $1 \%$ parasitaemia) for $72 \mathrm{~h}$ in a humidified atmosphere $\left(14 \% \mathrm{O}_{2}\right.$ and $\left.6 \% \mathrm{CO}_{2}\right)$ at $37{ }^{\circ} \mathrm{C}$, in 96-well flat bottom plates. Duplicate assays were performed for each sample. After incubation, $125 \mu \mathrm{L}$ supernatant was discarded and cells were washed twice with $125 \mu \mathrm{L} 1 \mathrm{X}$ PBS. $15 \mu \mathrm{L}$ re-suspended cells were transferred to 96-well flat bottom nonsterile black plates 
(Greiner Bio-one) already containing $15 \mu \mathrm{L}$ of the SYBR Green I lysis buffer (2X SYBR Green I, 20 mMTris base pH 7.5, 20 mM EDTA, 0.008\% w/v saponin, 0.08\% w/v Triton X100). Negative control, treated by solvents (DMSO or $\mathrm{H}_{2} \mathrm{O}$ ) and positive controls (chloroquine and doxycycline) were added to each set of experiments. Plates were incubated for $15 \mathrm{~min}$ at $37{ }^{\circ} \mathrm{C}$ and then read on a TECAN Infinite F-200 spectrophotometer with excitation and emission wavelengths at 485 and $535 \mathrm{~nm}$, respectively. The concentrations of compounds required to induce a $50 \%$ decrease of parasite growth $\left(\mathrm{IC}_{50} \mathrm{~K} 1\right)$ were calculated from three independent experiments.

\subsubsection{In vitro Cytotoxicity evaluation}

HepG2 cell line was maintained at $37{ }^{\circ} \mathrm{C}, 6 \% \mathrm{CO}_{2}, 14 \% \mathrm{O}_{2}, 80 \% \mathrm{~N}_{2}$, with $90 \%$ humidity in RPMI supplemented with 10\% fœtal bovine serum, 1\% L-glutamine (200 mM) and penicillin (100 U/mL) / streptomycin (100 $\mu \mathrm{g} / \mathrm{mL})$ (complete RPMI medium). The evaluation of the tested molecules cytotoxicity on the HepG2 (hepatocarcinoma cell line purchased from ATCC, ref HB-8065) cell line was performed according to the method of Mosmann [32] with slight modifications. Briefly, $5.10^{3}$ cells in $100 \mu \mathrm{L}$ of complete medium were inoculated into each well of 96-well plates and incubated at $37{ }^{\circ} \mathrm{C}$ in a humidified $6 \% \mathrm{CO}_{2}$. After $24 \mathrm{~h}$ incubation, $100 \mu \mathrm{L}$ of medium with various product concentrations dissolved in DMSO (final concentration less than $0.5 \% \mathrm{v} / \mathrm{v}$ ) were added and the plates were incubated for $72 \mathrm{~h}$ at $37{ }^{\circ} \mathrm{C}$. Triplicate assays were performed for each sample. Each plate-well was then microscopeexamined for detecting possible precipitate formation before the medium was aspirated from the wells. $100 \mu \mathrm{L}$ of MTT (3-(4,5-dimethyl-2-thiazolyl)-2,5-diphenyl-2 $H$-tetrazolium bromide) solution $(0.5 \mathrm{mg} / \mathrm{mL}$ in medium without FCS $)$ were then added to each well. Cells were incubated for $2 \mathrm{~h}$ at $37^{\circ} \mathrm{C}$. After this time, the MTT solution was removed and DMSO $(100 \mu \mathrm{L})$ was added to dissolve the resulting blue formazan crystals. Plates were shaken vigorously $(700 \mathrm{rpm}$ ) for $10 \mathrm{~min}$. The absorbance was measured at $570 \mathrm{~nm}$ with $630 \mathrm{~nm}$ as reference wavelength using a BIO-TEK ELx808 Absorbance Microplate Reader. DMSO was used as blank and doxorubicin (purchased from Sigma Aldrich) as positive control. Cell viability was calculated as percentage of control (cells incubated without compound). The $50 \%$ cytotoxic concentration $\left(\mathrm{CC}_{50}\right)$ was determined from the dose-response curve by using the TableCurve software $2 \mathrm{D}$ v.5.0. $\mathrm{CC}_{50}$ values represent the mean value calculated from three independent experiments. 


\section{Acknowledgement}

This work was supported by the Centre National de la RechercheScientifique (CNRS) and by Aix-Marseille Université (AMU).

\section{References}

[1] World Health Organization (WHO). World Malaria Report 2015. Geneva, Switzerland: World Health Organization (WHO),http://www.who.int/malaria/publications/world-malariareport-2015/report/en/

[2] A. P. Phyo, S. Nkhoma, K. Stepniewska, E. A. Ashley, S. Nair, R. McGready, C. L. Moo, S. Al-Saai, A. M. Dondorp, K. M. Lwin, P. Singhasivanon, N. P. J. Day, N. J. White, T. J. C. Anderson, F. Nosten, Emergence of artemisinin-resistant malaria on the west border of Thailand: a longitudinal study. Lancet 379 (2012) 1960-1966.

[3]M. D. Spring, J. T. Lin, J. E. Manning, P. Vanachayangkul, S. Somethy, R. Bun, Y. Se, S. Chann, M. Ittiverakul, P. Sia-ngam, W. Kuntawunginn, M. Arsanok, N. Buathong, S. Chaorattanakawee, P. Gosi, W. Ta-aksorn, N.Chanarat, S. Sundrakes, N. Kong, T. K.Heng, S. Nou, P. Teja-isavadharm, S. Pichyangkul, S. T. Phann, S. Balasubramanian, J. J. Juliano, S. R. Meshnick, C. M. Chour, S. Prom, C. A. Lanteri, C. Lon, D. L. Saunders, Dihydroartemisinin-piperaquine failure associated with a triple mutant including kelch13 C580Y in Cambodia: an observational cohort study. Lancet Inf. Dis. 15 (2015) 683-691.

[4] B. St Laurent, B. Miller, T. A. Burton, C. Amaratunga, S. Men, S. Sovannaroth, M. P. Fay, O. Miotto, R. W. Gwadz, J. M. Anderson, R. M. Fairhurst, Artemisinin-resistant Plasmodium falciparum clinical isolates can infect diverse mosquito vectors of Southeast Asia and Africa. Nat. Commun. 6 (2015) 8614.

[5] S. Zhu, J. Wang, G. Chandrashekar, E. Smith, X. Liu, Y. Zhang, Synthesis and evaluation of 4-quinazolinone compounds as potential antimalarial agents. Eur. J. Med. Chem. 45 (2010) 3864-3869.

[6] C. Mendoza-Martinez, J. Correa-Basurto, R. Nieto-Meneses, A. marquez-Navarro, R. Aguilar-Suarez, M. Dinora Montero-Cortes, B. Nogueda-Torres, E. Suarez-Contreras, N. Galindo-Sevilla, A. Rojas-Rojas, A. Rodriguez-Lezama, F. Hernandez-Luis, Design, 
synthesis and biological evaluation of quinazoline derivatives as anti-trypanosomatid and anti-plasmodial agents, Eur. J. Med. Chem. 96 (2015) 296-307.

[7] N. A. Malmquist, T. A. Moss, S. Mecheri, A. Scherf, M. J. Fuchter, Small-molecule histone methyltransferase inhibitors display rapid antimalarial activity against all blood stage forms in Plasmodium falciparum, Proc. Natl. Acad. Sci. 109 (2012) 16708-16713.

[8] S. Sundriyal, N. A. Malmquist, J. Caron, S. Blundell, F. Liu, X. Chen, N. Srimongkolpithak, J. Jin, S. A. Charman, A. Scherf, M. J. Fuchter, Development of diaminoquinazoline histone lysine methyltransferase inhibitors as potent blood-stage antimalarial compounds, ChemMedChem. 9 (2014) 2360-2373.

[9] A. Mishra, K. Srivastava, R. Tripathi, S. K. Puri, S. Batra, Search for new pharmacophores for antimalarial activity. Part III: synthesis and bioevaluation of new 6thioureido-4-anilinoquinazolines, Eur. J. Med. Chem. 44 (2009) 4404-4412.

[10] P. Verhaeghe, N. Azas, S. Hutter, C. Castera-Ducros, M. Laget, A. Dumètre, M. Gasquet, J.-P. Reboul, S. Rault, P. Rathelot, P. Vanelle, Synthesis and in vitro antiplasmodial evaluation of 4-anilino-2-trichloromethylquinazolines, Bioorg. Med. Chem. 17 (2009) 43134322.

[11] Y. Kabri, N. Azas, A. Dumètre, S. Hutter, M. Laget, P. Verhaeghe, A. Gellis, P. Vanelle, Original quinazoline derivatives displaying antiplasmodial properties, Eur. J. Med. Chem. 45 (2010) 616-622.

[12] S. Djekou, A. Gellis, J. Maldonado, M. P. Crozet, P. Vanelle, A radical synthetic approach to the new potentially bioactive pyrimidinones, Heterocycles 55 (2001) 535-544.

[13] P. Verhaeghe, P. Rathelot, S. Rault, P. Vanelle, Convenient preparation of original vinylic chlorides with antiparasitic potential in quinoline series, Lett. Org. Chem. 3 (2006) 891-897.

[14] N. Primas, P. Suzanne, P. Verhaeghe, S. Hutter, C. Kieffer, M. Laget, A. Cohen, J. Broggi, J-C. Lancelot, A. Lesnard, P. Dallemagne, P. Rathelot, S. Rault, P. Vanelle, N. Azas, 
Synthesis and in vitro evaluation of 4-trichloromethylpyrrolo[1,2-a]quinoxalines as new antiplasmodial agents, Eur. J. Med. Chem. 83 (2014) 26-35.

[15] P. Verhaeghe, N. Azas, M. Gasquet, S. Hutter, C. Ducros, M. Laget, S. Rault, P. Rathelot, P. Vanelle, Synthesis and antiplasmodial activity of new 4-aryl-2trichloromethylquinazolines, Bioorg. Med. Chem. Lett. 18 (2008) 396-401.

[16] P. Verhaeghe, A. Dumètre, C. Castera-Ducros, S. Hutter, M. Laget, C. Fersing, M. Prieri, J. Yzombard, F. Sifredi, S. Rault, P. Rathelot, P. Vanelle, N. Azas, 4-Thiophenoxy-2trichloromethyquinazolines display in vitro selective antiplasmodial activity against the human malaria parasite Plasmodium falciparum, Bioorg. Med. Chem. Lett. 21 (2011) 60036006.

[17] N. Primas, P. Verhaeghe, A. Cohen, C. Kieffer, A. Dumètre, S. Hutter, S. Rault, P. Rathelot, N. Azas, P. Vanelle, A New Synthetic Route to Original Sulfonamide Derivatives in 2-Trichloromethylquinazoline Series: A Structure-Activity Relationship Study of Antiplasmodial Activity, Molecules. 17 (2012) 8105-8117.

[18] C. Kieffer, P. Verhaeghe, N. Primas, C. Castera-Ducros, A. Gellis, R. Rosas, S. Rault, P. Rathelot, P. Vanelle, Sonogashira cross-coupling reaction in 4-chloro-2trichloromethylquinazoline series is possible despite a side dimerization reaction, Tetrahedron 69 (2013) 2987-2995.

[19] C. Castera-Ducros, N. Azas, P. Verhaeghe, S. Hutter, P. Garrigue, A. Dumètre, L. Mbatchi, M. Laget, V. Remusat, F. Sifredi, S. Rault, P. Rathelot, P. Vanelle, Targeting the human malaria parasite Plasmodium falciparum: In vitro identification of a new antiplasmodial hit in 4-phenoxy-2-trichloromethylquinazoline series, Eur. J. Med. Chem. 46 (2011) 4184-4191.

[20] A. R. Genady, promising carboranylquinazolines for boron neutron capture therapy: synthesis, characterization and in vitro toxicity evaluation, Eur. J. Med. Chem. 44 (2009) 409-416. 
[21] R. A. Smits, I. J. P. de Esch, O. P. Zuiderveld, J. Broeker, K. Sansuk, E. Guaita, G. Coruzzi, M. Adami, E. Haaksma, R. Leurs, Discovery of quinazolines as histamine H4 receptor inverse agonists using a scaffold hopping approach, J. Med. Chem. 51 (2008) 78557865.

[22] A. Purohit, R. Benetti, M. Hayes, M. Guaraldi, M. Kagan, P. Yalamanchili, F. Su, M. Azure, M. Mistry, M. Yu, S. Robinson, D. D. Dischino, D. Casebier, Quinazoline derivatives as MC-I inhibitors: evaluation of myocardial uptake using positron emission tomography in rat and non-human primate, Bioorg. Med. Chem. Lett. 17 (2007) 4882-4885.

[23] T-A. N. Pham, Z. Yang, Y. Fang, J. Luo, J. Lee, H. Park, Synthesis and biological evaluation of novel 2,4-disubstituted quinazoline analogues as GPR119 agonists, Bioorg. Med. Chem. 21 (2013) 1349-1356.

[24] M. Spulak, J. Pourova, M. Voprsalova, J. Mikusek, J. Kunes, J. Vacek, M. Ghavre, N. Gathergood, M. Pour, Novel bronchodilatoryquinazolines and quinoxalines: synthesis and biological evaluation, Eur. J. Med. Chem. 74 (2014) 65-72.

[25] A. Gellis, C. Kieffer, N. Primas, G. Lanzada, M. Giorgi, P. Verhaeghe, P. Vanelle, A new DMAP-catalyzed and microwave-assisted approach for introducing heteroarylamino substituents at position 4 of the quinazoline ring, Tetrahedron 70 (2014) 8257-8266.

[26] Y. Kabri, P. Verhaeghe, A. Gellis, P. Vanelle, Regioselective Suzuki-Miyaura Reaction: application the microwave-promoted synthesis of 4,7-diarylquinazolines, Molecules 15 (2010) 2949-2961.

[27] H-Y Li, Y. Wang, W. T. McMillen, A. Chatterjee, J. E. Toth, S. R. Mundla, M. Voss, R. D. Boyer, J. Scott Sawyer, A concise synthesis of quinazoline TGF-b RI inhibitor through one-pot three-component Suzuki-Miyaura/etherification and imidate-amide rearrangement reactions, Tetrahedron 63 (2007) 11763-11770.

[28] P. Verhaeghe, P. Rathelot, A. Gellis, S. Rault, P. Vanelle, Highly efficient microwave assisted $\alpha$-trichlorination reaction of $\alpha$-methylated nitrogen containing heterocycles, Tetrahedron. 62 (2006) 8173-8176. 
[29] D. B. Jordan, G. S. Basarab, D-I. Liao, W. M. P. Johnson, K. N. Winzenberg, D. A. Winkler, Structure-based design of inhibitors of the rice blast fungal enzyme trihydroxynaphtalene reductase, J. Mol. Graphics Modell. 19 (2001) 434-447.

[30] W. Trager, J.B. Jensen, Human malaria parasites in continuous culture, Science. 193 (1976) 673-675.

[31] W.A. Guiguemde, A.A. Shelat, D. Bouck, S. Duffy, G.J. Crowther, P.H. Davis, D.C. Smithson, M. Connelly, J. Clark, F. Zhu, M.B. Jiménez-Díaz, M.S. Martinez, E.B. Wilson, A.K. Tripathi, J. Gut, E.R. Sharlow, I. Bathurst, F. El Mazouni, J.W. Fowble, I. Forquer, P.L. McGinley, S. Castro, I. Angulo-Barturen, S. Ferrer, P.J. Rosenthal, J.L. Derisi, D.J. Sullivan, J.S. Lazo, D.S. Roos, M.K. Riscoe, M.A. Phillips, P.K. Rathod, W.C. Van Voorhis, V.M. Avery, R.K. Guy, Chemical genetics of Plasmodium falciparum, Nature. 465 (2010) 311315.

[32] T. Mosmann, Rapid colorimetric assay for cellular growth and survival: application to proliferation and cytotoxicity assays, J. Immunol. Methods. 65 (1983). 
Graphical Abstract: 